\title{
UNE NÉCROPOLE DU BAS-EMPIRE AUX MARTRES-D’ARTIÈRES (Puy-de-Dôme)
}

\author{
par Robert PERICHON et Claude CHOPELIN
}

Le bourg des Martres-d'Artières est situé à proximité de l'Allier, à quelques kilomètres au nord de Pont-du-Château. La nécropole a été découverte à l'est de l'intersection que forment la route de Pont-du-Château à Maringues et celle, plus petite, qui conduit de cette dernière au bourg des Martres (fig. 1)1.

Une indication plus précise peut être donnée par la carte E. M. au $1: 80000^{\mathrm{e}}$ : la découverte se localise à proximité de la cote 312, au lieu-dit Puy-Redon. De nombreuses cxploitations de sable ouvrent de béantes carrières dans les terrasses alluviales qui, en cet endroit, dominent l'Allier de quelques dizaines de mètres. Actuellement, le site archéologique a complètement disparu dans l'exploitation de la gravière (fig. $2, \mathrm{n}^{0} 1$ ). Il est cependant possible que des zones non exploitées, à l'ouest de la route de Pont-du-Château à Maringues, recèlent encore des vestiges.

C'est en 1964 que la découverte a été effectuée : les propriétaires du terrain, désireux d'ouvrir une carrière de sable, firent décaper à l'aide d'engins mécaniques la couche de terre de culture superficiclle variant de 50 à $70 \mathrm{~cm}$. C'est dans cette couche que furent observés, parmi de petits mais nombreux fragments de céramiques rouges (sigillées ?), des vases communs intacts disposés près de sépultures ou de lambeaux de ces dernières. Il s'agissait d'inhumations simples; les squelettes reposaient en pleine terre, sans qu'une construction en brique ou en pierre signale leur présence. Les quelques clous recueillis ne permettent pas de supposer la présence de cercueils utilisés systématiquement. Quelques fragments de calottes craniennes ont été conservés. Les vestiges les plus abondants sont les céramiques.

1 Au cours de l'été 1968, M. Aimé Rudel, professeur agrégé à l'E. N. de Clermont-Ferrand, nous informait d'une découverte de vestiges céramiques en bordure de l'Allier. Obligeamment, il nous mettait en relation avec les inventeurs : MM. Ducroix père et fils qui nous montrèrent leurs découvertes. Devant leur importance, nous décidâmes d'en effectuer l'analyse et le catalogue. Nous ne saurions trop remercier M. A. Rudel ainsi que $M$. et $M^{\text {me }}$ Ducroix qui ont lout fait pour faciliter notre travail et qui conservent précieusement les collections que nous décrivons. Toutes les informations sur la découverte nous ont été communiquées par leurs soins. De plus, nous devons à M. Ducroix fils une restauration soignée des pièces brisées. Que tous veuillent bien trouver ici l'expression de notre vive gratitude. 


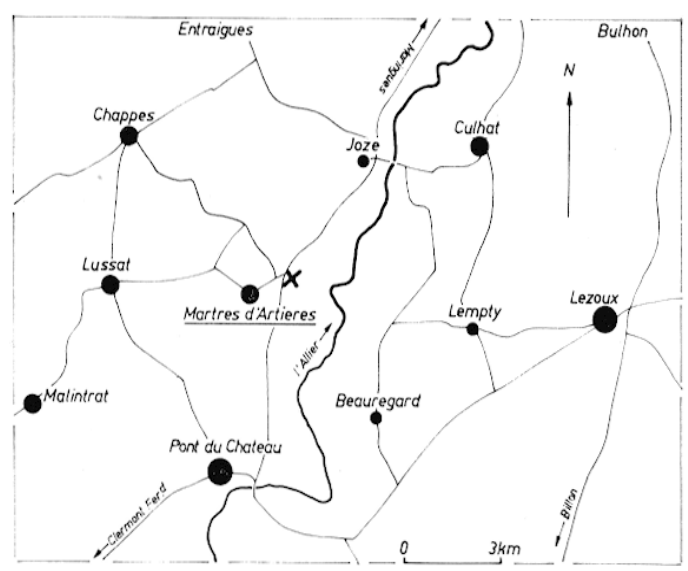

1 Le site des Martres-d'Artières. L'emplacement des découvertes est marqué par une croix.
Les terres de culture dans laquelle ces vestiges ont été exhumés recouvrent une basse lerrasse de l'Allier caractérisée par une faune à Elephas Primigenius ${ }^{2}$ dont une dent remarquable a été exhumée dans la même exploitation ${ }^{3}$.

La fig. $2, \mathrm{n}^{0} 2$, montre la couche de terre de culture reposant sur les alluvions.

Les vestiges exhumés sont essentiellement des céramiques, à part quelques clous, un fer de lance ${ }^{4}$ et quelques restes osseux humains.

\section{Les céramiques.}

Nous distinguons deux catégories selon une méthode qui, de prime abord, peut sembler curieuse : d'une part, les céramiques très fragmentées et, d'autre part, les vases intacts ou présentant des traces de cassures récentes et se reconstituant facilement.

a) Les céramiques fragmentées. Elles consistent en quelques poteries communes mais les plus intéressantes, en même temps que les plus importantes, sont les céramiques sigillées qu'il a été possible de dater avec une certaine précision (fig. 3). La production de ces céramiques couvre approximativement le $\mathrm{II}^{\mathrm{e}}$ siècle ; la majeure partie provient des officines de Lezoux, peu éloignées. Les motifs ou les styles appartiennent aux productions des potiers ARCANUS, BUTRIO, CASURIUS et DONNAUCUS, tous ayant œuvré dans le Centre de la Gaule. Les plus anciens étaient en activité au début du ${ }_{11}{ }^{\mathrm{e}}$ siècle et les plus récents, en particulier CASURIUS, sous l'empereur Commode. Il a été observé que ces fragments de céramiques ne se trouvaient pas en corrélation avec les vases de la nécropole ; ils semblent appartenir à une phase d'occupation antérieure à cette dernière.

b) Les vases intacts ou se reconstituant facilement. Ils se présentent sous deux aspects. Le premier, le plus abondant, consiste en vases communs, d'usage domestique courant. Le second consiste en quelques petits vases colorés cherchant à imiter la céramique sigillée. Les céramiques domestiques communes se répartissent en deux grands groupes de formes : hautes et basses. Les formes hautes comprennent des vases ovoïdes plus ou moins écrasés, des vases à col haut et des pichets à une seule anse parfois munie d'un poussier. On note également des flacons à liquides à goulot étroit. Les formes basses comprennent

2 H. Pelletier, Les Limagnes d'Auvergne, édit. G. de Bussac, Clermont-Ferrand, 1969. Les terrasses inférieures sont également caractérisées par Rhinocéros tichorinus, Rangifer lerandus, Equus caballus, Bison priscus, Cervus elaphus, Félis priscus, elc... (cf. P. Lapado, Hargues et A. Ruder, Notice explicative de la carte géologique.).

3 Collection Ducroix.

4 Découvert à une centaine de mèlres de la nécropole. Rien ne prouve cependant que cette pièce soit contemporaine. 


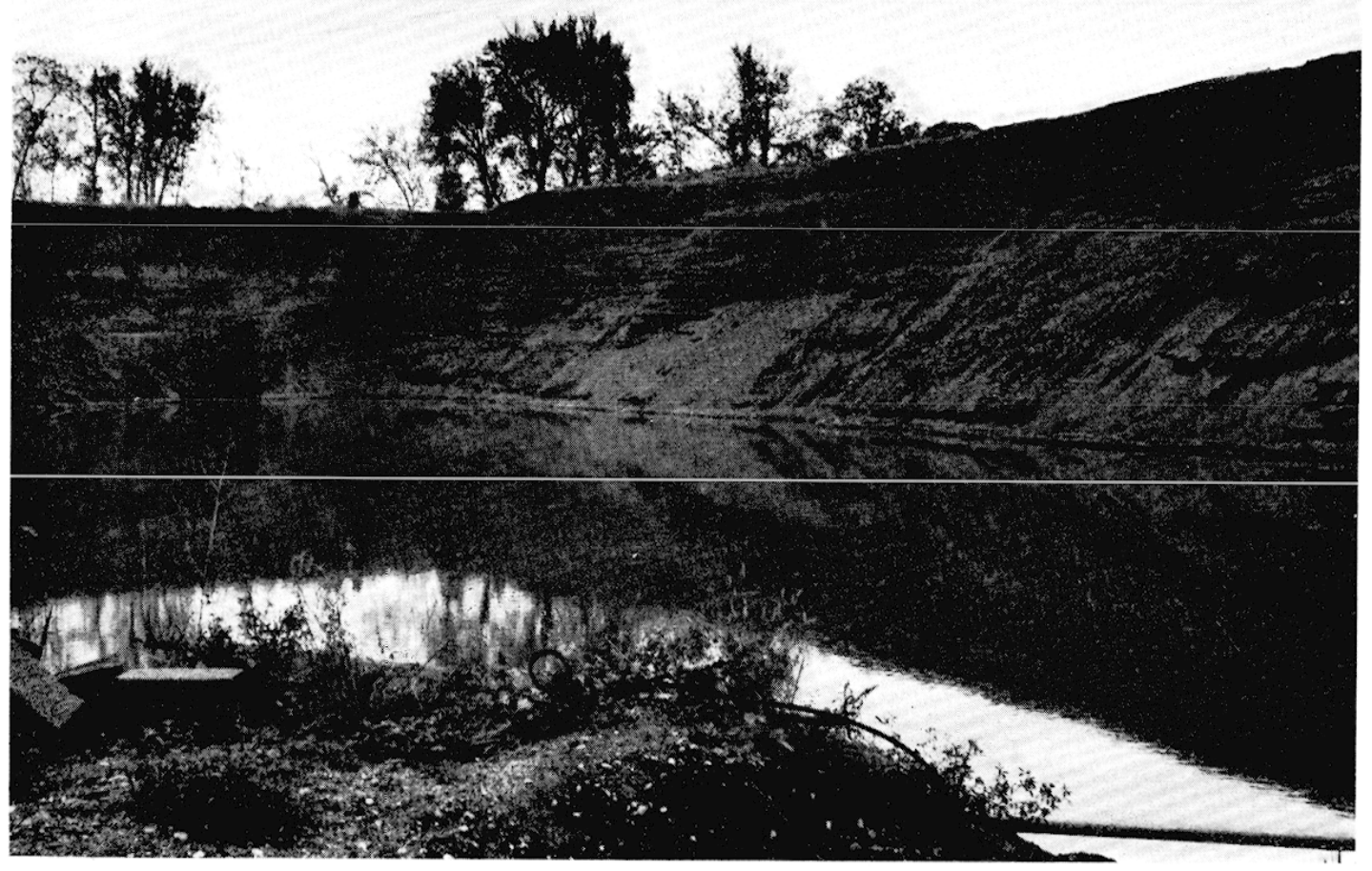

2

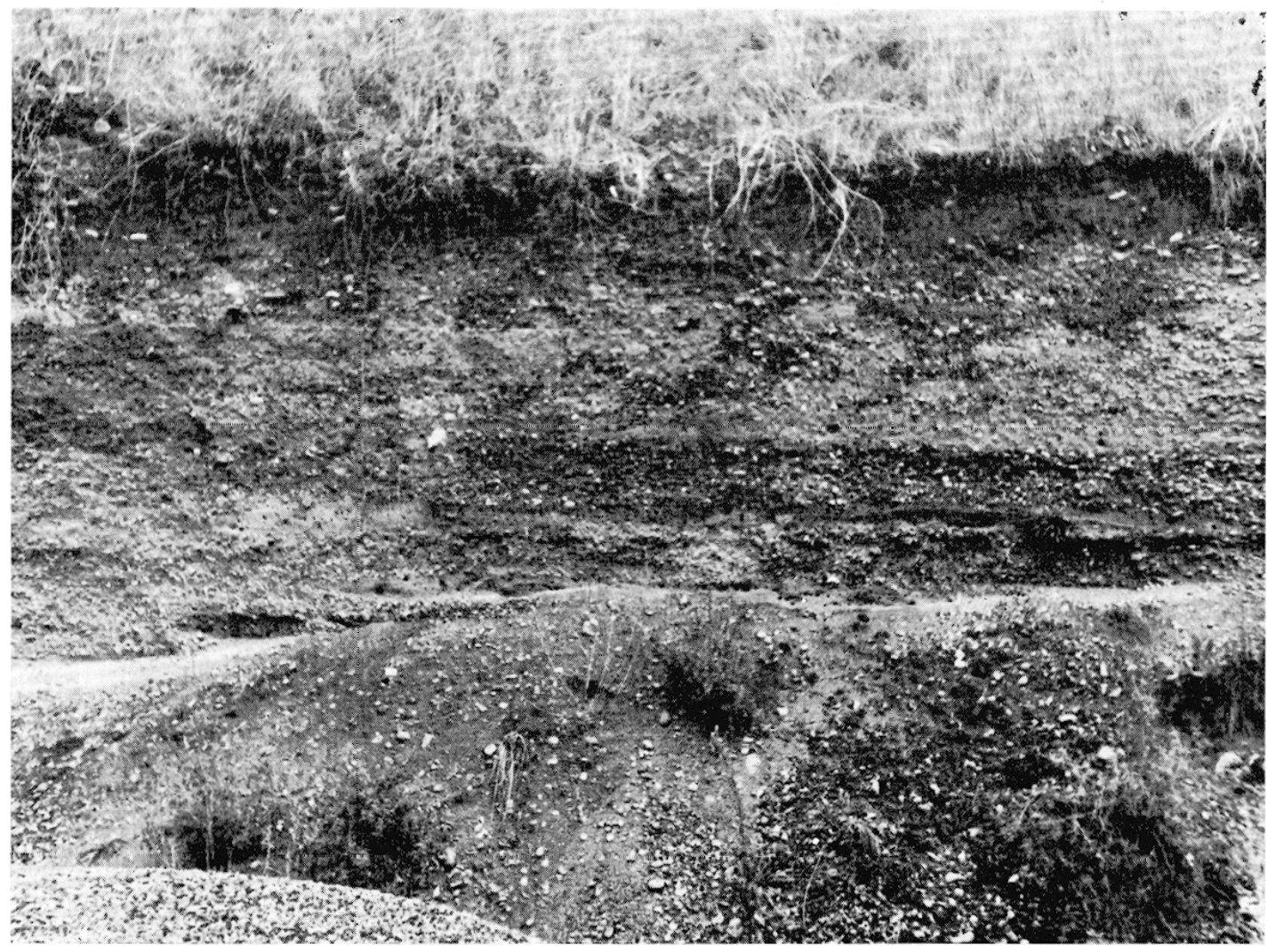

2 1. La carrière Ducroix. - 2. Stratigraphic. Couche d'humus reposant sur la terrasse alluviale. 
des marmites tripodes, des coupes plus ou moins carénées et des écuelles munies de collerettes. Des écuelles plus simples sont ornées de cannelures : ces cannelures sont d'ailleurs l'un des rares éléments décoratifs que présente l'ensemble de cees céramiques.

\section{Le verre.}

Peut-être était-il plus abondant ? Un seul exemplaire nous est parvenu, à peu près entier (fig. 17, $\mathrm{n}^{0} 33$ ). Il s'agit d'un gobelet dont le type semble se rencontrer fréquemment

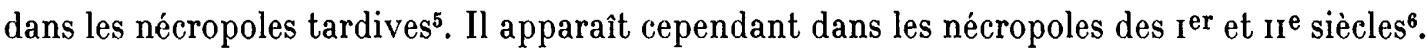

\section{Les vestiges métalliques (fig. 10 ).}

Ils se réduisent à quelques clous grossiers, très oxydés. L'objet le plus important reste un fer de lance assez bien conservé, emmanché par une solide douille et dont le profil montre qu'il s'agit d'un travail soigné. Il est difficile d'attribuer une date précise de fabrication à cet objet.

$$
* *
$$

Les lieux-dits "les Martres " indiquent souvent l'emplacement d'une nécropole. Cette appellation viendrait du mot "martyr" et aurait pour origine la découverte de restes humains? ${ }^{7}$ A. Vincent proposait de donner au mot Martre le sens de martyr, corps, reste de martyrs, lieu ou ces restes ont été trouvés. En fait, Martre désigne d'antiques nécropoles souvent situées le long des voies romaines ${ }^{8}$. Ce toponyme, répandu en Auvergne" ${ }^{9}$, le semble encore davantage dans le Sud de la Bourgogne, plus particulièrement dans le Clunisois ${ }^{10}$. Martre ne désigne pas obligatoirement un site chrétien : des nécropoles païennes ont souvent pu être assimilées à des sépultures de martyrs ${ }^{11}$. Une étude exhaustive de ces toponymes serait sans doute pleine d'intérêt.

Dans quel contexte se situe la nécropole des Martres-d'Artières ? Nous possédons un certain nombre d'éléments de comparaisons : d'autres nécropoles ont livré des vestiges très comparables à ceux que nous présentons. La plus proche est celle de la Maison-Blanche (commune de Pardines, Puy-de-Dôme), bien décrite par P.-F. Fournier ${ }^{12}$. Cette nécropole

5 Par exemple en Lorraine, dans les fouilles de Scraponne (cf. Informations de la circonscriplion de Lorraine, dans Gallia, XXVI, 1966, p. 375), ou encore dans la nécropole de Barisis-aux-Bois (cf. B. LAGrorx, La nécropole du IV e siècle de Barisis-aux-Bois, dans Gallia, XII, 1954, nos 14, 15, 16 et 17 de la figure 6).

6 Cf. A. Grange, H. Parriat et R. Perraud, La nécropole de Briord (Ain), dans Revue Physiophile de Montceau-les-Mines, $\mathrm{n}^{\circ} 52,1960$, p. 42 et 43 , fig. 7/24.

7 G. Fournier, Le peuplement rural en Basse Auvergne durant le Haut Moyen Age, publ. de la Faculté des Lettres de Clermont-Ferrand, $2^{\text {e }}$ série, XII, 1962.

8 A. Vincent, Toponymie de la France, Bruxelles, 1937, p. 307 : l'Auteur fait allusion au Manuel de Graxitr (II partie, l'archéologie du sol) dans lequel on trouve une étude du mot Martres.

9 G. Fournier, Le peuplement..., p. 421.

10 Document manuscrit de F. Jarreau, auquel nous exprimons nos remerciements; d'après une étude de E. Magnen. En Forez, le toponyme répandu est Martoret, Martorey, Martouret, Martourcy. La forme "Martres " est inexistante (cf. J. E. Dufour, Dictionnaire topographique du Forez, Måcon, 1946).

11 Ce qui semble être le cas de la nécropole des Martres-d'Artières.

12 E. Desforges et P.-F. Fournier, La nécropole de la Maison Blanche (commune de Pardines), dans Revue d'Auvergne, 1945, t. 59. 
a livré des céramiques très voisines de celles observées aux Martres-d'Artières : en particulier des jattes domestiques communes ${ }^{13}$, des petites coupes en terre sigillée $e^{14}$ recouvertes d'un enduit rouge orangé ou rouge brun, mat, peu épais et très détérioré. Ces dernières ne présentent aucun décor et peuvent être comparées à celles figurées sous nos nos 28 à 31 , (fig. 8). Le site de la Maison-Blanche a également livré un gobelet en verre dont la description laisse supposer qu'il est très proche de celui que nous figurons sous le $\mathrm{n}^{0} 33$ (fig. 8). Une nécropole, très éloignée de l'Auvergne, celle de Barisis-au-Bois, étudiée et datée par M. l'abbé Lacroix, présente des vestiges très voisins de ceux des Martres ${ }^{15}$. Il en est de même pour ceux exhumés dans la nécropole de Briord (Ain), fouillée par H. Parriat ${ }^{16}$.

Roanne (Rodumna), pauvre en vestiges du Bas-Empire, possède cependant, révélée par des travaux récents, une fosse à incinération de cette époque dans laquelle on observe certaines céramiques relativement proches de celles que nous étudions ${ }^{17}$. Toujours à Roanne, dans une nécropole étudiée par Joseph Déchelette ${ }^{18}$, des sépultures ont livré des céramiques du Bas-Empire dont plusieurs sont proches de celles des Martres ${ }^{19}$. Les mêmes types céramiques sont observés dans des sépultures de même époque découvertes dans la vallée du Rhône $e^{20}$.

Il n'est pas toujours possible de faire état d'une liaison entre les nécropoles et l'habitat : en effet, pour celles que nous venons d'évoquer, nous ne connaissons que bien partiellement les habitats correspondants et dans la plupart des cas ils n'ont jamais été découverts. Cependant, il est important de noter la similitude qui existe entre le mobilier rencontré dans les sépultures et celui de certains habitats tel celui observé à Saint-Maurice-lèsChâteauneuf (Saône-et-Loire) ${ }^{21}$, celui de Jœuvre22 et d'une façon encore plus nelle dans celui du Iv siècle découvert aux Fontaines-Salées et dont l'inventaire descriptif a été publié par M. l'abbé Lacroix ${ }^{23}$.

Il est curieux de constater, en se plaçant sur un plan différent, la similitude qui existe entre les céramiques du Bas-Empire que l'on découvre dans le Centre de la Gaule et celles observées en Rhénanie ${ }^{24}$. Cette similitude est-elle suffisante pour évoquer le fait d'une colonisation germanique voulue, dans le but de repeupler certaines régions ?25. Les décou-

13 Ibid., p. 16, fig. XVI, no 115.

14 Ibid., p. 14, fig. XV, nos 161 et 166 .

15 Abbé B. LaGroix, La nécropole de Barisis-aux-Bois (Aisne), dans Gallia, XII, 1954, fig. 5.

16 A. Grange, H. Parriat et R. Perraud, La nécropole..., p. 43, fig. 21 et 24.

17 P. Vernat et J. Poncet, Une fosse à incinération gallo-romaine, dans Bulletin des Groupes de Recherches archéologiques du Département de la Loire, 1968/69, p. 19 à 36, pl. 4, fig. 1, pl. 5, fig. 1 et pl. 6, fig. 1 .

18 J. Déchelette, La nécropole gallo-romaine de Roanne, dans Bulletin de La Diana, XII, 1904.

19 L. Grelaud, Les sépultures Rollet, dans les Cahiers d'archéologie régionale du Groupe de recherches archéologiques de Roanne, no 5, déc. 1966., pl. 1, fig. 1 et 3.

20 S. GAGNì̀re, Les sépultures à inhumalion du $I I I^{\mathrm{e}}$ au XIII siècles de notre ère dans la basse vallée du Rhône, dans les Cahiers Rhodaniens, XII, 1965, p. 67, fig. 13 et 14.

21 Cl. Chopelin et J. Quey, Le sile gallo-romain de La Tour à St-Maurice-lès-Chaleauneuf, dans Revue archéologique du Centre, VIII, 1, 1969, p. 14. 1965.

22 R. Pŕrichon et Cl. Chopein, La céramique à décor esiampé de Joeuvre (Loire), dans Ogam, XVII, 3-6,

23 Abbé B. Lacrorx, Mobilier d'un habitat du IV e siècle aux Fontaines-Salees, Inventaire descriptif, dans Revue archéologique de l'Est, XIX, 1-4, 1968, p. 197, fig. 11, p. 198, fig. 12 et 13, p. 202, fig. 21, 22 et 23, p. 230 , fig. 48.

24 E. Gose, Gefasstypen der römischen Keramilk im Rheinland, 1950.

25 P. Vernat et J. Poncet, Une fosse..., p. 28. 
vertes sont encore trop clairsemées et plus le fait du hasard que dues à une analyse systématique pour que nous puissions émettre autre chose qu'une hypothèse.

J.-J. Hatt suppose que la sculpture qui caractérise la décoration des tombeaux - inexistants dans le cas des Martres - est inséparable du milieu artisanal et commerçant ${ }^{26}$. Et, dans l'ensemble complexe et disparate de ces monuments, l'art qui nous est montré ne serait teinté d'aucun spiritualisme ${ }^{27}$. Ne découvririons-nous ce spiritualisme qu'au travers des modestes offrandes des tombes sans stèles ? Que signifie précisément l'absence de stèles dans la nécropole des Martres-d'Artières ?28 Est-elle l'indice d'une pauvreté certaine ou sommes nous en présence de groupes ethniques particuliers se refusant à des usages d'origine romaine ? P.-M. Duval suppose que la diversite des types de tombes ne résulte pas seulement de la différence des conditions sociales et précise que les pierres tombales inscrites sont souvent le fait de petites gens ${ }^{29}$. Il est cependant vraisemblable que ces petites gens subissent les appauvrissements successifs que connait le Bas-Empire depuis Marc-Aurèle et voient leurs conditions de vie modifiées. Et, si le culte des morts subsiste, son aspect se modifie et l'embellissement des tombes n'est plus celui de la période de grande prospérité de l'Empire. J.-J. Hatt souligne d'ailleurs la prédominance, dans ce domaine, des facteurs économiques ${ }^{30}$.

L'extrême simplicité qui se dégage de la nécropole des Martres-d'Artières mérite d'être soulignée. En même temps, elle nous offre un éventail typologique, principalement en ce qui concerne la céramique.

\section{INVENTAIRES}

I. Céramiques sigillées appartenant à un contexte antérieur à celui de la nécropole (fig. 3).

$36^{31}$. - Fragment de rebord d'un vase de forme $37^{32}$. La technique en est assez grossière et nous sommes probablement en présence de motifs surmoulés. On distingue Vulcain (?) tenant une pièce de forgeron. Ce motif a été utilisé par le potier Drusus ${ }^{33}$, ainsi que par Arcanus $^{34}$.

37. - Fragment de vase 37 de Dragendorff. Il s'agit d'un travail assez soigné. Le relief des motifs est particulièrement accusé. On distingue un personnage marchant à droite, nu, parfaitement dessiné. Il semble correspondre au motif 638 d'Oswald ${ }^{35}$ et 344 de Déchelette ${ }^{36}$. P. Karnitsche

26 Cette sculpture connaît son apogée vers 150 (cf. J.-J. Hatr, La lombe gallo-romaine, P.U.F., 1951, p. 246).

27 Ibid., p. 247.

28 Malgré toutes nos investigations, nous n'en avons pas observé un seul fragment. Cette absence est également notoire à Roanne, tant dans le site de Gilbertèes (cf. P. Vernat et J. Poncer, Une fosse...) que dans la nécropole traditionnelle décrite par J. Décheletre (cf. op. cil., note 18).

29 P.-M. Duval, La vie quolidienne en Gaule, Paris, 1953, p. 321.

30 J.-J. HatT, La tombe gallo-romaine, p. 247.

31 Pour plus de simplicilé el pour éviter toute erreur d'identification, les numéros de références correspondent à celui de notre inventaire.

32 Pour les numéros de formes des céramiques sigillées, nous nous référons, sauf indication contraire, à la classification de DRAGENDORFF (cf. Terra Sigillata, dans Bonner Jahrbücher, XCVI-XCVII, 1895).

33 Voir J. A. Stanfield et G. Simpson, Cenlral Gaulish Potters, Londres, 1958, pl. 88, no 1/7.

34 Information de $\mathrm{H}$. Vertet, qui a bien voulu revoir nos déterminations et auquel nous exprimons nos vifs remerciements.

35 F. Oswald, Index of figure-types on Terra sigillata, 1936.

36 J. DÉchelette, Les vases ornés de la Gaule romaine, II, 1901. 


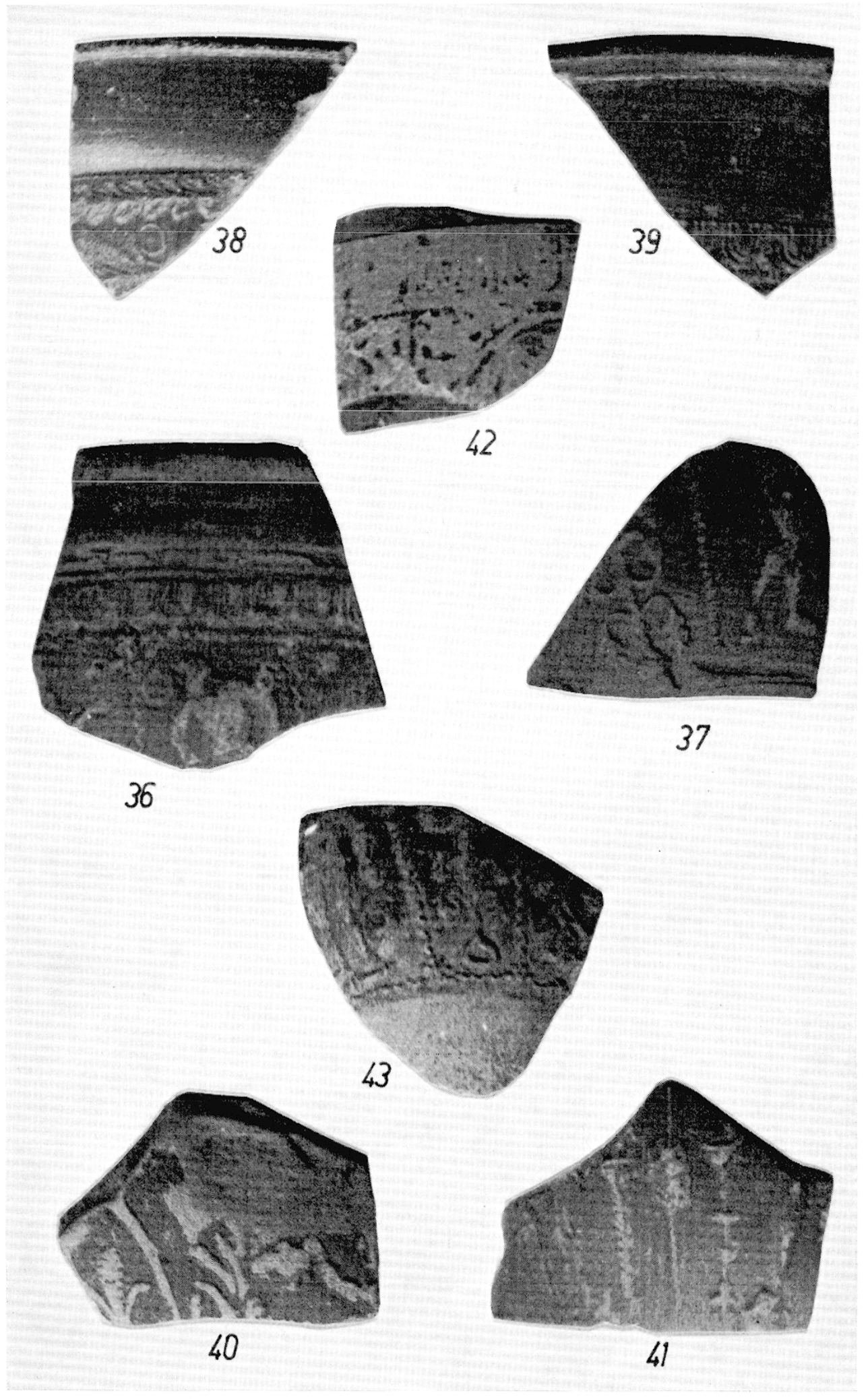

3 Les céramiques sigillées fragmentées, vases moulés. Nota : le nº 40 est inversé. 
l'attribue aux productions de Casurius $^{37}$. J. A. Stanfield et G. Simpson situent également ce motif dans les productions du Centre de la Gaule sous les Antonins ${ }^{38}$. Un motif en forme d'arbre stylisé figure sur ce même tesson. Il est fréquent dans l'art de Casurius. On le retrouve associé au personnage marchant à droite sur le fragment $\mathrm{n}^{\circ} 18, \mathrm{pl} .133$ de Stanfield et Simpson et isolé sur les fragments 15 et 17 de la même planche. Le potier Doeccus (Doiici) a également utilisé le motif du personnage marchant à droite ${ }^{39}$. Cinnamus et Avitus ont utilisé le motif en forme d'arbre stylisé. Il semble cependant que les motifs que nous venons de présenter appartiennent à l'art de Casurius. Ils sont présentés dans des métopes séparés par des rangs de perles.

38. - Fragment de rebord de forme 37 de Dragendorff présentant un décor de feuilles. Il s'agit d'une céramique très brillante, probablement une production du Centre de la Gaule, sous Trajan ${ }^{40}$.

39. - Fragment de partie supérieure de vase 37. La couverte est brillante. Seules les oves sont visibles $^{41}$, soulignées par un rang de perles.

40. - Fragment de panse de vase 37 . Le décor est difficile à interpréter. On distingue, à gauche du fragment, le bras d'un personnage; au centre un animal hypothétique el, à droite, un décor de feuilles. Il s'agit probablement d'une production de Lezoux, du II $^{\mathrm{e}}$ siècle.

41. - Fragment de panse de vase 37. Ce tesson présente des décors soignés. A gauche du tesson on distingue les jambes d'un personnage qu'une colonne torse sépare d'un petit masque ${ }^{42}$. A droite du masque, on observe une petite colonne, puis la base d'une colonne assez large. Il pourrait s'agir d'une production de Butrio ${ }^{43}$.

42. - Fragment de panse de vase 37 dont l'ornementation est très abîmée ce qui rend les motifs difficiles à identifier. Oves et motifs permettent cependant de supposer une production de Lezoux de la seconde moitié du ri ${ }^{\mathrm{e}}$ siècle $\mathrm{e}^{44}$.

43. - Fragment de panse de vase 37. Il s'agit d'une production soignée. Le relief des motifs est très accusé. On distingue au centre un arbre stylisét5 dans un métope déterminé par des lignes ondées; des fleurettes marquent les intersections de ces lignes. Drusus utilise ce motif, mais dans des métopes séparés par des rangs de perles ${ }^{46}$. Selon G. Simpson, ce motif est caractéristique de Drusus ${ }^{47}$. A gauche de ce motif est un personnage debout, tenant dans sa main gauche un masque dans un ovale. Drusus a également utilisé ce motif ${ }^{48}$. A droite du premier motif, on distingue une branche de vigne ${ }^{49}$. H. Vertet pense cependant qu'il peut s'agir d'une production du potier Arcanus $^{50}$.

43 bis (fig. 8). - Fragment de petite coupe du type 46 de Dragendorff. Le grésage est très brillant, rouge vif. La pâte, extrêmement fine est d'un rouge assez sombre. Il s'agit vraisemblablement d'une production du Sud de la Gaule ${ }^{51}$.

37 P. Karnitsche, Die Relief Sigillata von Ovilava, Linz, 1959, pl. 63, p. 204.

38 J. A. Stanfield et G. Simpson, Central..., pl. 133, personnage marchant à droite, sur les vases nos 14,18 , 20 et 21 .

39 Ibid., pl. 148, n 25, le personnage et le nom du potier sont dans le même métope.

40 Information de $\mathrm{H}$. Vertet.

41 Probablement oves de Donnaucus, période de Trajan.

42 Probablement le motif $1337 a$ d'Oswald (Index...).

43 Information de $\mathrm{H}$. Vertet, sa lettre du 19 février 1969.

44 Ibid.

45 Motif 1116 de Déchelette (Vases ornés...).

46 J. A. Stanfield et G. Simpson, Central..., pl. 88, fig. 3, 7 et 8.

47 Ibid., p. 156.

48 Ibid., pl. 50, n$^{\circ} 593$.

49 Ibid., motif de Drusus, p. 157.

50 Son information personnelle du 19 février 1969.

51 Ce type de vase a également été fabriqué dans le Centre de la Gaule (cf. Collection Constancias, au musée J. Déchelette, à Roanne). 
46 (fig. 9). - Fragment de partie supérieure d'assiette ou de plat dont l'aspect s'apparente a celui de certaines productions italiques ${ }^{52}$ mais son grésage et sa couleur nous le font attribuer au productions du Sud de la Gaule.

47. - Petit fragment de vase Dragendorff 29, à décor moulé. Un tendrill binding est visible ${ }^{53}$ et pourrait faire attribuer la fabrication du vase dont est issu ce fragment aux productions du Sud de la Gaule.

48 (fig. 9). - Fragment de mortier à rebord retombant. Une toute petite partie du déversoir est visible. Cette forme est caractéristique des productions du $\mathrm{II}^{\mathrm{e}} \operatorname{siècle}^{54}$.

\section{Céramiques communes ${ }^{55}$.}

1 (fig. 4 et 11). - Vase de forme haute. La panse est sphérique. Le col, tronconique, est très haut, légèrement évasé et, se termine par une lèvre en bourlet. Le pied, nettement détaché de la panse, se termine par un fond étroit et plat. La pâte est mi-fine. La coloration extérieure est d'un gris assez sombre; la pâte elle même est gris-clair. La surface extérieure présente de nombreuses traces de lissage. On observe aucune trace de décoration.

2 (fig. 4 et 11). - Gruche ansée. La panse est émisphérique mais, dans sa partie supérieure, présente des creux et des saillies dont la plus haute est à angle vif. L'intersection du col et de la panse est marquée par un bourlet. Le col, très étroit, est en forme de bobine. Sa partie la plus étroite est marquée par deux cannelures. Une lèvre assez grossière, et partiellement brisée, le termine. Le pied, étroit, se détache de la panse et se termine par un fond plat. Les anses partent de la partie supérieure de la panse et se rattachent à la lèvre du col ; elles sont courbes et celle qui est restée fixée au récipient montre, dans sa partie supérieure, une sorte d'ergot. La pâte est mi-fine, d'une couleur rouge assez sombre qui témoigne d'une cuisson à feu oxydant. Un engobe blanc est disposé sur toute la surface du récipient. Par-dessus cet engobe, deux bandes horizontales d'une peinture rouge-orangé occupent les creux de la panse. Cette couleur, passée au pinçeau, semble caractéristique de l'ornementation peinte tardive $e^{56}$. Il est possible de faire un rapprochement entre ce vase et ceux d'une série à peu près identique découverts à Lezoux ${ }^{57}$. Les décors peints ne sont pas tout à fait les mêmes; les formes sont proches cependant et les anses des vases de Lezoux présentent la même particularité de posséder un ergot à la courbure ${ }^{58}$.

3 (fig. 4 et 11). - Flacon de forme haute. La panse, ovoïde, se prolonge et se rétrécit pour former le col qui se présente sous la forme d'un évasement tronconique. Une seule anse, fine, part de la partie supérieure de la panse pour se terminer en dessous du col. Le pied, tronconique, présente un fond plat. La pâte, mi-fine, est rose et recouverte d'un engobe la faisant ressembler à de la céramique sigillée. Cet engobe est recouvert de trois lignes horizontales blanches disposées dans la partie la plus renflée de la panse.

52 Loeschake, Keramische Funde und Hallern, 1909, pl. 10.

53 OsWALD, Index of Figure-types..., pl. LXXXV.

54 Oswald et Pryce, An Introduction to the Sludy of Terra Sigillata, 1920, pl. 73.1, et E. Gose, Gefässtypen..., p. 14 et pl. $9, \mathrm{n}^{\circ} 147$.

55 Les dimensions des récipients ne figurent pas dans les descriptions, ce type d'information étant donné par les planches de dessins et les photographies.

56 R. PÉrichon, La céramique peinte dans la plaine du Forez..., dans Celticum IX, 1963, p. 162, vase du groupe G, pl. 1 et 2 .

57 J.-J. Hatt et P.-F. Fournier, Vases à décor peint découverts à Lezoux, dans Bul. historique et scientifique de l'Aulergne, I.XII, 1942, no 516, n. 214.

58 Ibid., "Cruche no 3 , le coude supérieur de l'anse est marqué par une sorte d'ergot " et, note 6, * une autre anse, qui n'a pu être recollée... présente le même ergot à la courbure supérieure ». 


$$
\left\{\begin{array}{l}
58 \\
8
\end{array}\right.
$$


4 (fig. 4 et 11). - Flacon bas. L'aspect est peu élégant. La panse est arrondie, très large et se rétrécit rapidement pour former un col particulièrement étroit, peu détaché au-dessus de la panse. La lèvre forme un gros bourlet triangulaire, s'achevant par une arête vive. Le fond plat ne se détache pas de la panse. Une anse courbe, assez large, relie la panse à la partie inférieure de la lèvre du col. Lors du tournassage, le pied a été détaché du tour à la ficelle comme l'indiquent les traces visibles sur le fond. La pâte est rougeâtre mais la surface du récipient semble avoir été recouverte d'une sorte d'engobe marron-clair.

5 (fig. 4 et 12). - Gobelet. Ce récipient à panse ovoïde est incomplet : le col manque. L'aspect général doit se rapprocher de celui du vase $n^{0} 1$. Le pied se détache nettement de la panse; il est bitronconique et se termine par un fond plat. La panse est ornée de sept dépressions disposées dans la partie la plus renflée. La surface est noirâtre. Il peut s'agir d'un engobe car la pâte est d'un gris assez clair. Cette pâte, mi-fine, a tendance à s'écailler. En surface, on observe quelques traces de mica.

6 (fig. 4 et 12). - Pelil vase ovoïde. La panse est presque hémisphériquc. Lc col, tronconique, s'en détache par une arête vive et se termine par un évasement. Le pied, assez étroit, se détache également de la panse. Il est en couronne ; sa forme est tronconique. Le fond présente en son centre un ombilic assez prononcé. La pâte, mi-fine, est grise. La surface du récipient est presque noire et soigneusement lissée.

7 (fig. 5 et 12). - Vase ovoïde. La panse est régulière, émisphérique; elle s'évase pour former le col qui se termine par une lèvre épaisse. Le pied, étroit, ne se détache pas de la panse. La pâte est beige clair. Grossière, elle présente de gros grains de quartz qui apparaissent en surface. Il semble qu'un engobe a été passé sur la surface extérieure ce qui donne au vase un aspect marron.

8 (fig. 5 et 12). - Vase ovoïde. L'aspect en est écrasé. La panse est sphérique et s'évase pour former le col qui se termine par une petite lèvre triangulaire à arête vive. Le pied se détache nettement de la panse et sa partie la plus large est également marquée par des arêtes vives. Le fond est incurvé et présente, en son centre, une sorte de pastille en relief (fig. $5, \mathrm{n}^{0} 8 \mathrm{a}$ ). La pâte est grossière, grise et le récipient présente en surface de nombreuses imperfections. Le seul élément de décor est une rainure horizontale dans la partie supérieure de la panse.

8 bis (fig. 6). -- Vase ovoïde très fragmenté. La panse sphérique est plus ou moins régulière. Le col s'en détache par une arête vive ; il s'évase légèrement et se termine par une lèvre triangulaire assez épaisse. Le pied est étroit ; il se détache de la panse par un petit tronc de cône. Le fond est plat, légèrement incurvé. La pâte est grossière, rougeâtre et témoigne d'une cuisson en atmosphère oxydante. La surface extérieure est rose et, par endroits, marron. Le seul élément de décor est une rainure horizontale dans la partie supérieure de la panse.

9 (fig. 5 ct 12). - Vase ovoïde haut. La panse est émisphérique dans sa partie supérieure mais elle s'allonge vers le bas. Le col, assez large, est séparé de la panse par une arête vive; il se termine par une petite lèvre en bourlet. Le pied, tronconique, ne se détache pas de la panse et s'achève par un fond plat. La pâte est assez grossière, rougeâtre. La surface extérieure, de couleur marron, semble avoir été recouverte d'une couche de mica, L'ensemble est soigneusement lissé.

10 (fig. 5 et 12). - Vase ovoïde haut. La panse est émisphérique dans sa partie supérieure mais elle s'allonge vers le bas. Le col, assez large, est séparé de la panse par une arête vive; il se termine par une petite lèvre en bourlet légèrement débordante. Le pied, tronconique mais très peu évasé, se termine par un fond plat. La pâte est mi-fine, claire à l'intérieur. Les surfaces sont légèrement brunes ; il semble qu'elles aient été recouvertes d'une couche de mica.

11 (fig. 5 et 13). - Pichet. La panse est sphérique. Le col, tronconique, est séparé de la panse par une arête vive. Il est haut et se termine par un court évasement qu'achève une petile lèvre triangulaire. Le pied s'évase et se détache nettement de la panse. Le fond est concave. L'anse courbe, 

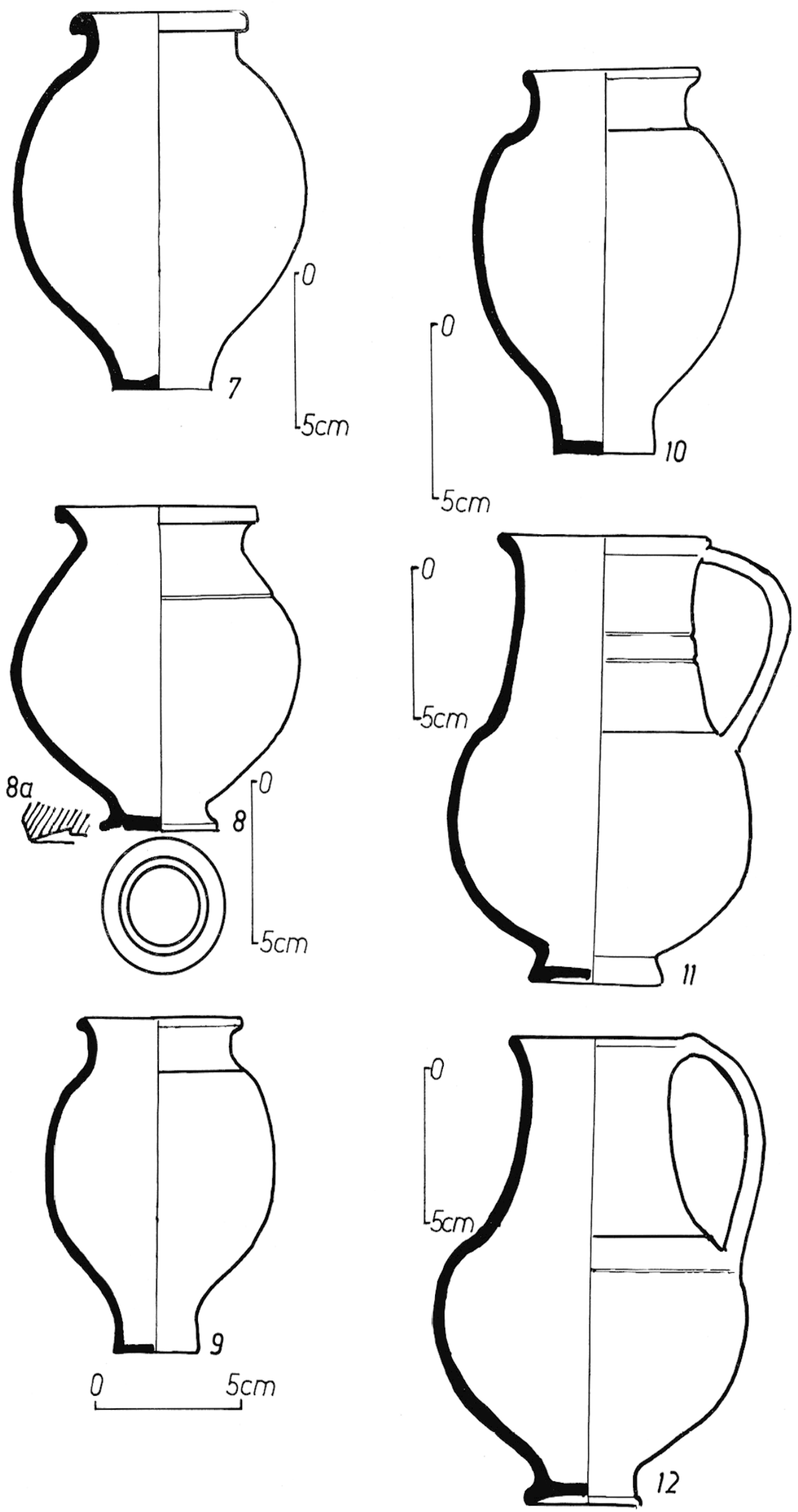

5 Céramiques communes, vases d'offrandes de la nécropole. Cf. fig. 12, les photographies des vases nos 7 à 10 et, fig. 13, celles des vases nos 11 et 12 . 
assez épaisse, part de l'intersection du col et de la panse et se soude à la lèvre du col. La pâte est grossière; la cassure brechoïde. L'intérieur du récipient présente un montage assez grossier. Les surfaces sont soigneusement lissćes mais de gros grains de quartz sont apparents. L'ensemble est de couleur beige-clair mais des traces de feu l'assombrissent. Trois rainures horizontales ornent la partie supérieure de la panse et le col.

12 (fig. 5 et 13 ). - Pichet. La panse est sphérique. Le col, tronconique, est séparé par une arête vive de la panse. Il est haut et se termine par un court évasement qu'achève un bourlet arrondi. Le pied, en forme de bourlet, se termine par un fond légèrement concave. L'anse est arrondie. Fixée à la partie la plus renflée de la panse, elle se termine à la partie supérieure du col. La pâte, mi-fine, présente quelques traces de mica. Les parois sont épaisses, ce qui donne au récipient un poids considérable par rapport à ses dimensions. L'intérieur de la pâte est d'un beige assez clair mais l'ensemble du récipient, y compris l'intérieur du col, est peint d'une couleur rouge assez sombre, passée au pinceau. Une cannelure décore la partie supérieure de la panse.

13 (fig. 6 et 13). - Gobelet de forme haute. La panse est sphérique. Le col tronconique est très haut; légèrement évasé, il se termine par une lèvre formant un petit bourlet. Le pied se détache bien de la panse, prend une forme tronconique et s'achève par un fond plat dans lequel une cannelure circulaire a été incisée près de la périphérie. La pâte est mi-fine, assez grossière cependant, et de couleur marron sombre. Le vase est soigneusement lissé et semble recouvert d'un engobe noir el brillant. Ce genre de céramique à la particularité de s'écailler par larges plaques, ce que montre parfaitement notre photographie $\mathrm{n}^{0} 13$, fig. 13.

14 (fig. 6 et 13). - Pelil vase ovoïde. La panse, sphérique, s'allonge vers le bas. Une arête vive, précède une couronne horizontale d'où émerge un col évasé se terminant par une lèvre peu prononcée. Le pied, peu saillant se termine sur un fond plat. Sur la surface du vase, les traces de tournassage sont très visibles. I a pâte, assez fine, est grisâtre, tendre. La cuisson, peu régulière, montre des traces de coup de feu. La surface extérieure est particulièrement rugueuse.

15 (fig. 6 et 13). - Petit pichet. La panse est ovoïde. Le col en est bien détaché par une arête vive. Il se présente sous la forme d'une grosse bobine incurvée et s'achève, dans sa partie supérieure par une lèvre arrondie un petit peu plus épaisse que la paroi. Le pied, tronconique, est saillant. Il s'achève par un fond plat marqué par une cannelure près de la périphérie. A l'intérieur, un petit ombilic marque le milieu du fond. L'anse, brisće, part de la panse et vient se souder à la partie supérieure du col. La pâte est mi-fine, gris-clair dans la cassure, elle est un peu plus sombre en surface; la cuisson semble asse\% rustique. Une cannelure orne la partie supérieure de la panse.

16 (fig. 6 et 14). - Coupe ou écuelle de forme basse. Le récipient est composé de deux parties principales : la panse, qui se présente sous la forme d'une large courbe et, un flanc, légèrement rentrant, qui se termine par une lèvre en bourlet. L'intersection entre le flanc et la panse est marquée par une saillie en forme de bourlet. Le pied est creux et le fond apparemment plat. La pâte est fine, d'un gris assez clair. Des traces de tournassage sont, malgré le lissage, parfaitement visibles sur toute la surface du récipient.

17 (fig. 6 et 14). - Coupe ou écuelle de forme basse. On distingue deux parties principales : la panse, sous la forme d'une large coupe et un flanc à peu près vertical, se terminant par un bourlet un peu déversé sur l'extérieur. L'intersection du flanc et de la panse est marquée par un petit bourlet saillant. Le pied est en couronne mais le fond du récipient prolonge la courbe de la panse. La pâte, mi-fine, est gris-clair. Un engobe de couleur gris sombre enrobe le récipient.

18 (fig. 6 et 14). - Marmite tripode. L'écuelle, légèrement carénée se termine par une lèvre en bourlet soulignée par une cannelure. Le fond est en cuvette. Les pieds ne sont que de simples pustules rapportées plus ou moins adroitement après le tournassage. La pâte est grossière ; la cuisson semble régulière. L'ensemble est gris. 

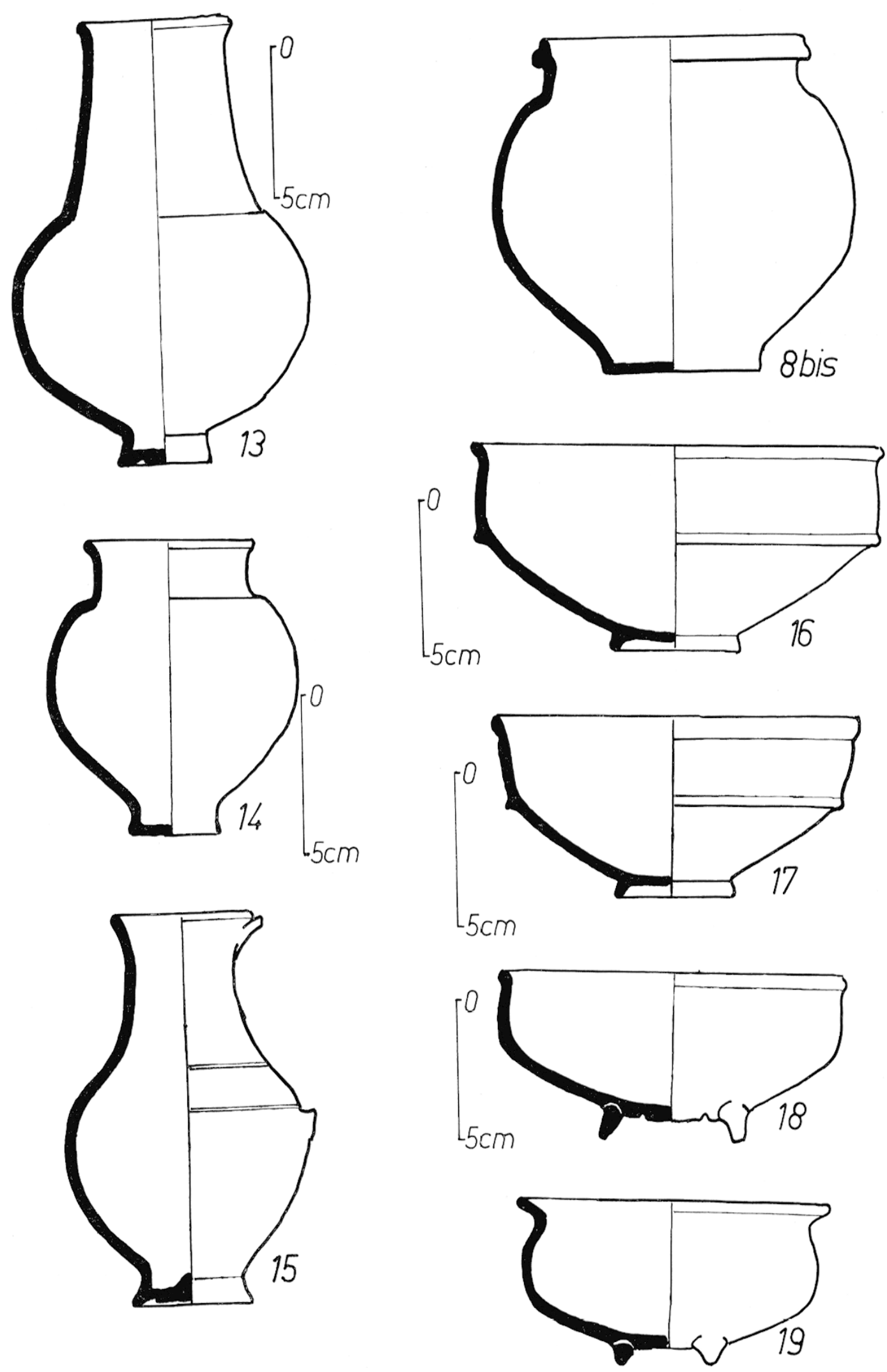

6 Céramiques communes, vases d'offrandes de la nécropole. Cf. fig. 13, les photographies des vases nos 13 à 15 et, fig. 14, celles des vases 16 à 19 . 
19 (fig. 6 et 14). - Petite marmite tripode. La partie supérieure de l'écuelle est un peu rentrante et se termine par une lèvre évasée de forme rectangulaire. La pâte est mi-fine; la cuisson oxydante.

20 (fig. 7 et 14). - Coupe basse. La partie supérieure s'évase légèrement et se termine par une arête assez fine. Un rebord horizontal, dont le profil est rectangulaire, forme une sorte de collerette à quelques millimètres en dessous de la lèvre. Le pied, tronconique, est soudé au fond de l'écuelle et partiellement évidé par une rainure triangulaire. L'intérieur de la pâte est gris; la cuisson est réductrice. Les surfaces sont noires ; un engobe a probablement été utilisé pour donner cette coloration.

21 (fig. 7 et 14). - Coupe basse. La panse forme une large courbe qui va, se refermant, vers le haut. La séparation entre la partie évasée et la partie refermée de la panse est marquée par une collerette dont le profil est triangulaire. Le rebord supérieur du récipient est marqué, à l'extérieur, par une petite rainure. Le fond prolonge la courbe de la panse et un pied en couronne, légèrement tronconiquc, supporte le récipient. La pâte est mi-fine, d'un gris jaunâtre dans son contexte ; la cuisson est réductrice. Les surfaces sont noires. La matière s'écaille et se clive facilement.

22 (fig. 7 et 15). - Coupe haute. La panse se présente sous la forme d'une large courbe continue jusqu'au rebord supérieur. Vers les deux tiers supérieurs de la panse est soudée une collerette au profil retombant. Le fond prolonge la courbe de la panse. Le pied est en couronne, tronconique et, marqué par une petite rainure intérieure, concentrique à la couronne. La céramique est grossière. L'intérieur de la pâte est gris. Les surfaces sont noires.

23 (fig. 7 et 15). - Coupe basse. La panse forme une large courbe qui va, se refermant, vers le haut. La séparation entre la partie évasée et la partie refermée de la panse est marquée par une collerette légèrement retombante dont le profil est triangulaire. Le rebord supérieur du récipient est souligné, à l'extérieur, par une petite rainure horizontale. Le fond prolonge la courbe de la panse et un pied en couronne, légèrement tronconique, supporte le récipient. La fabrication est assez soignée. La pâte est grise à l'intérieur ; les surfaces sont noires.

24 (fig. 7 et 15). - Coupe basse. La panse forme une large courbe qui va se refermant vers le haut. La séparation entre la partie évasée et la partie refermée de la panse est marquée par une petite collerette dont le profil est triangulaire. Le rebord supérieur du récipient est marqué, à l'extérieur, par une cannelure assez large, disposée horizontalement. Le fond intérieur du récipient est dans le prolongement de la courbe de la panse ; le fond extérieur forme un petit ombilic. Un pied en couronne dont le profil est triangulaire termine le récipient. Pâte et surfaces sont grises. L'extérieur est assez soigné.

25 (fig. 7 et 16). - - Coupe carénée. La panse, partant directement de la base du récipient, forme une large courbe peu incurvée ; aux deux tiers supérieurs, elle se referme brusquement pour enfin s'évaser vers le rebord supérieur. Ce dernier est arrondi et son épaisseur est la même que celle de la panse. A l'intersection des deux mouvements on observe un petit redan au profil triangulaire, situé dans le prolongement extérieur de sa partie refermée. Le récipient ne possède pas de pied et se termine par un fond plat, un peu saillant. La pâte est mi-fine, grise à l'intérieur. Les surfaces sont également grises.

26 (fig. 7 et 16). - Grande coupe à rebord rentrant. Le col, qui est dans le prolongement de la courbe de la panse, souligné par deux fortes cannelures horizontales, présente un profil plus épais que cette dernière. Le pied, anguleux, tronconique, est en couronne. La partie interne du fond est plate mais, la face extérieure du fond, à l'intérieur du pied, présente un petit ombilic. Des traces de mica sont visibles sur l'ensemble de la surface extérieure du récipient. La pâte est mi-fine, soigneusement lissée. La cuisson est oxydante. 

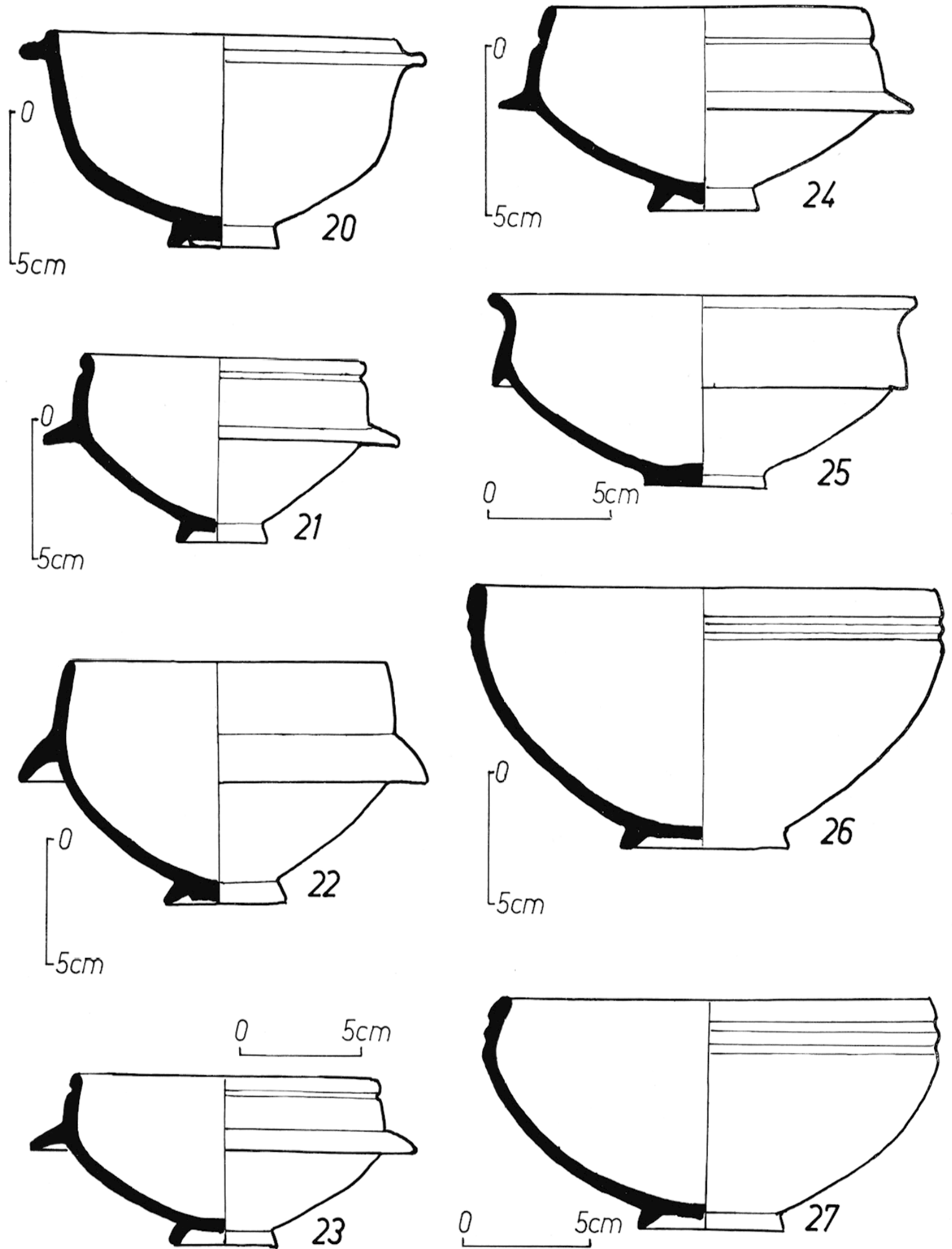

7 Céramiques communes, vases d'offrandes de la nécropole. Cf. fig. 14, les photographies des vases nos 20 et 21 ; fig. 15 , celles des vases nos 22,23 et 24 ; fig. 16, celles des vases nos 25,26 et 27 . 
27 (fig. 7 et 16). - Grande coupe à rebord rentrant. Le col, qui est dans le prolongement de la courbe de la panse, souligné par deux cannelures horizontales, présente un profil plus épais que cette dernière. Le pied, en couronne, est tronconique. La pâte est mi-fine. La cuisson est oxydante mais sa coloration est claire, blanchâtre. Il semble que pour palier à l'irrégularité de la coloration des surfaces el'es ont été enduites, à l'éponge, d'une couleur ocre-rouge. Le fond, épais, se termine en ombilic à l'extérieur.

28 (fig. 8 et 16). - Imitation de céramique sigillée ou céramique sigillée de fabrication locale. Il s'agit d'une petite coupe légèrement évasée, munie d'un pied tronconique court. Le fond se termine extérieurement par un ombilic. Deux petites rainures, l'une près de la lèvre, l'autre dans le milieu de la panse, décorent l'extérieur du récipient. Revenant à la panse, il est important d'observer que sa courbe n'est pas régulière : à peu près à mi-hauteur, elle forme un angle déterminant une sorte de carène. Cette disposition, même sur de petits récipients, est caractéristique des vases de fabrication tardive. Le fait a été bien observé par Oswald ${ }^{59}$ et Gose réunit un certain nombre d'informations identiques recueillies sur un vaste territoire ${ }^{60}$. La pâte, relativement fine, est d'une coloration rosâtre assez soutenue. Elle est assez tendre peut-être en raison d'une cuisson effectuée à température peu élevée $^{61}$. En surface, il semble que nous ne soyons plus en présence d'un grésage de la pâte comme c'est le cas pour la plupart des sigillées de Lezoux, par exemple, mais d'une sorte d'enduit peu solide dont seules quelques traces subsistent.

29 (fig. 8 et 16). - Petite coupe imitant la céramique sigillée. La panse est carénée, légèrement évasée. Le pied est tronconique, en couronne, peu élevé. Le fond prolonge la courbe de la panse sans former, à l'extérieur, d'ombilic prononcé. Une seule rainure décore l'extérieur du vase. La pâte est fine et rose. Sur les surfaces, l'enduit, mal passé, se présente sous des couleurs variables qui vont du rouge au noir. Des traces de doigts abondantes sont visibles sur toute la surface.

30 (fig. 8 et 16). - Petite coupe imitant la céramique sigillée. La panse est carénée, légèrement évasée. Le fond et le pied manquent. Une rainure décore l'extérieur de la panse. La pâte est fine et rose. L'enduit des surfaces, mal passé, varie du rouge au noir.

31 (fig. 8 et 17). - Petite coupe imitant la céramique sigillée. La courbe de la panse est continue ; elle se referme légèrement vers le haut. Une petite rainure horizontale marque à peu près la mihauteur de cette panse. Le pied est tronconique, en couronne. Le fond se situe dans le prolongement de la courbe de la panse. Sa surface extérieure est marquée par deux petites gorges concentriques. Cette pièce est un peu plus soignée extérieurement que les vases 29 et 30. La pâte est fine, rose. L'enduit est rouge sombre.

32 (fig. 8 et 17). - Petit vase ovoïde, de forme écrasée. La panse s'allonge vers le bas. Dans sa partie supérieure, elle se rétrécit pour former le col qui, à partir de là, s'évase. Une lèvre triangulaire termine ce col. Elle est marquée par des arêtes vives. La surface du récipient présente des traces assez marquées de tournassage. La pâte est mi-fine, la cuisson oxydante. Des traces de mica sont apparentes en surface et dans la masse. L'ensemble est de couleur beige-clair.

34 (fig. 8). - Col de flacon à liquides. La lèvre se présente, extérieurement, en forme de poulie. Le départ d'une anse est visible. La pâte est fine, oxydée. La surface extérieure est recouverte d'un engobe blanc.

44 (fig. 8 et 17). - Gruche à deux anses. Le col manque. La panse, en toupie, est formée de deux troncs de cônes opposés, réunis par une brève courbe. Les départs d'anses sont bien visibles sur la partie supérieure de la panse. Le fond est plat et montre des traces de découpage au fil. La pâte est

59 Oswat.n, Introduction..., pl. 48 , nos 10 et 16.

60 Gefässlypen..., pl. 2, fig. 20 , pl. 3 , fig. 27 et 29 .

61 Par comparaison avec la dureté des pâtes des céramiques sigillées produites antérieurement. 

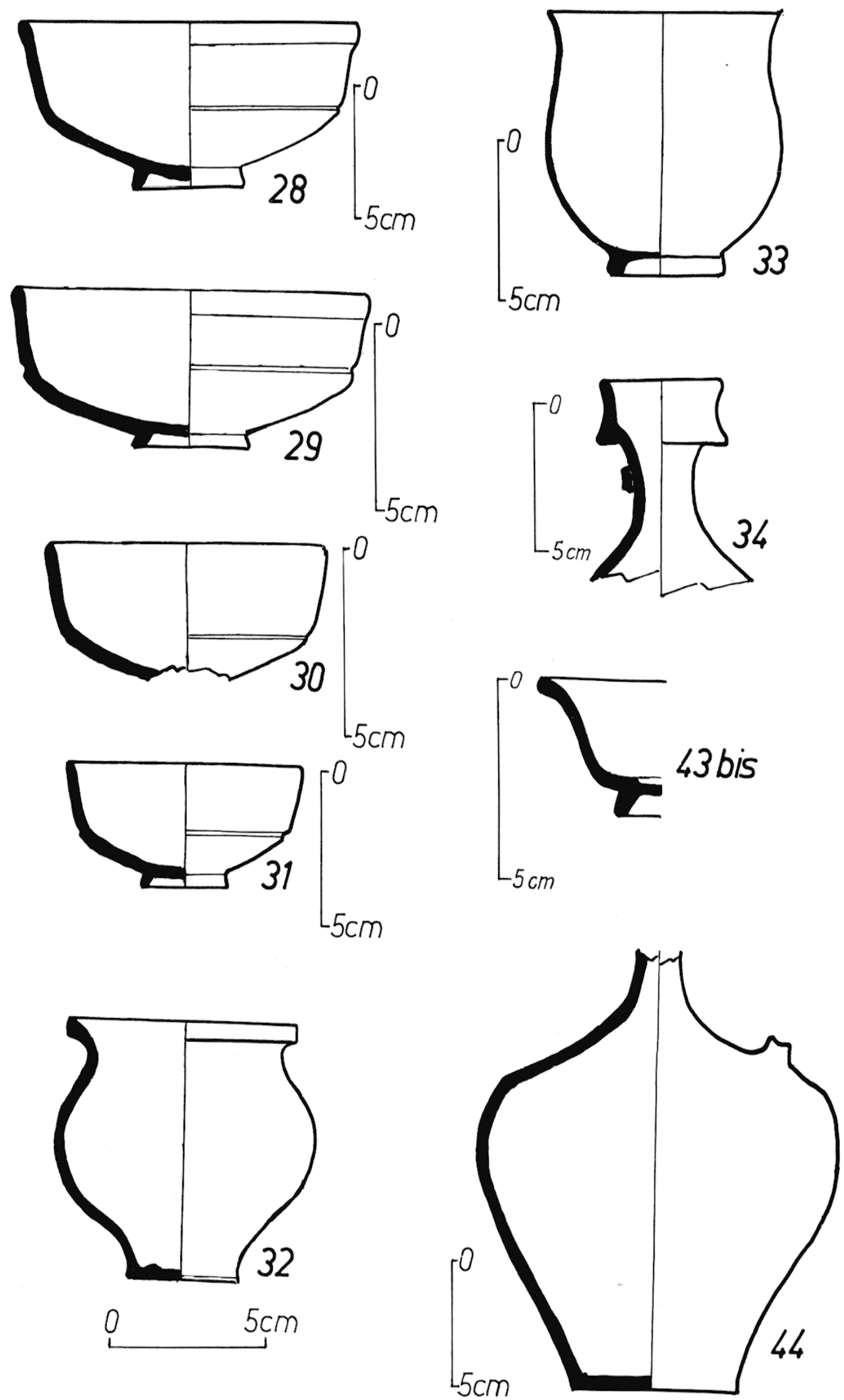

8 Vases d'offrandes de la nécropole. Les vases nos 28 à 31 sont des imitations de céramiques sigillées dont les photographies se trouvent fig. 16 et 17 . Les photographies des vases 32,33 (verre) et 44 , sont fig. 17 . Les vases nos 34 et 43 bis appartiennent à un contexte antérieur à la nécropole. 

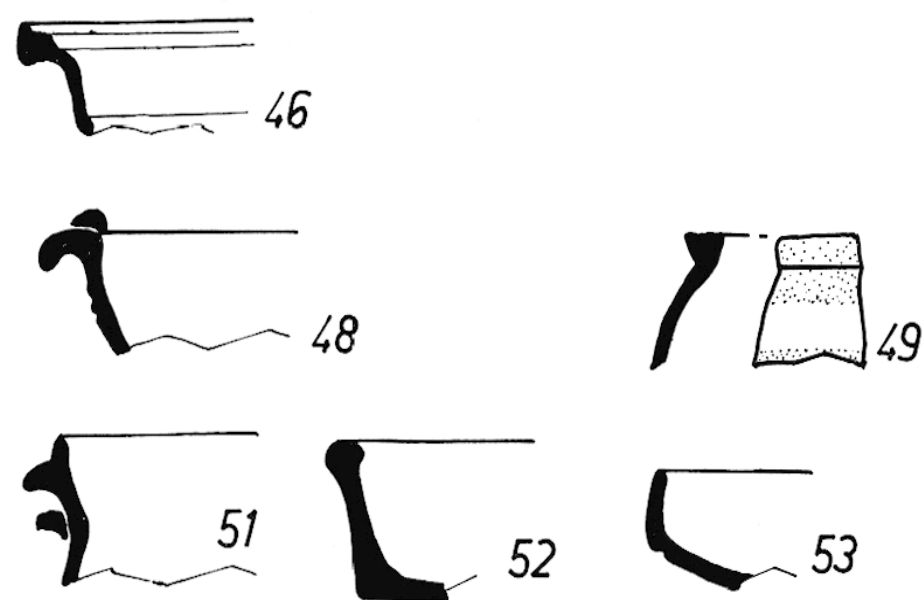

52
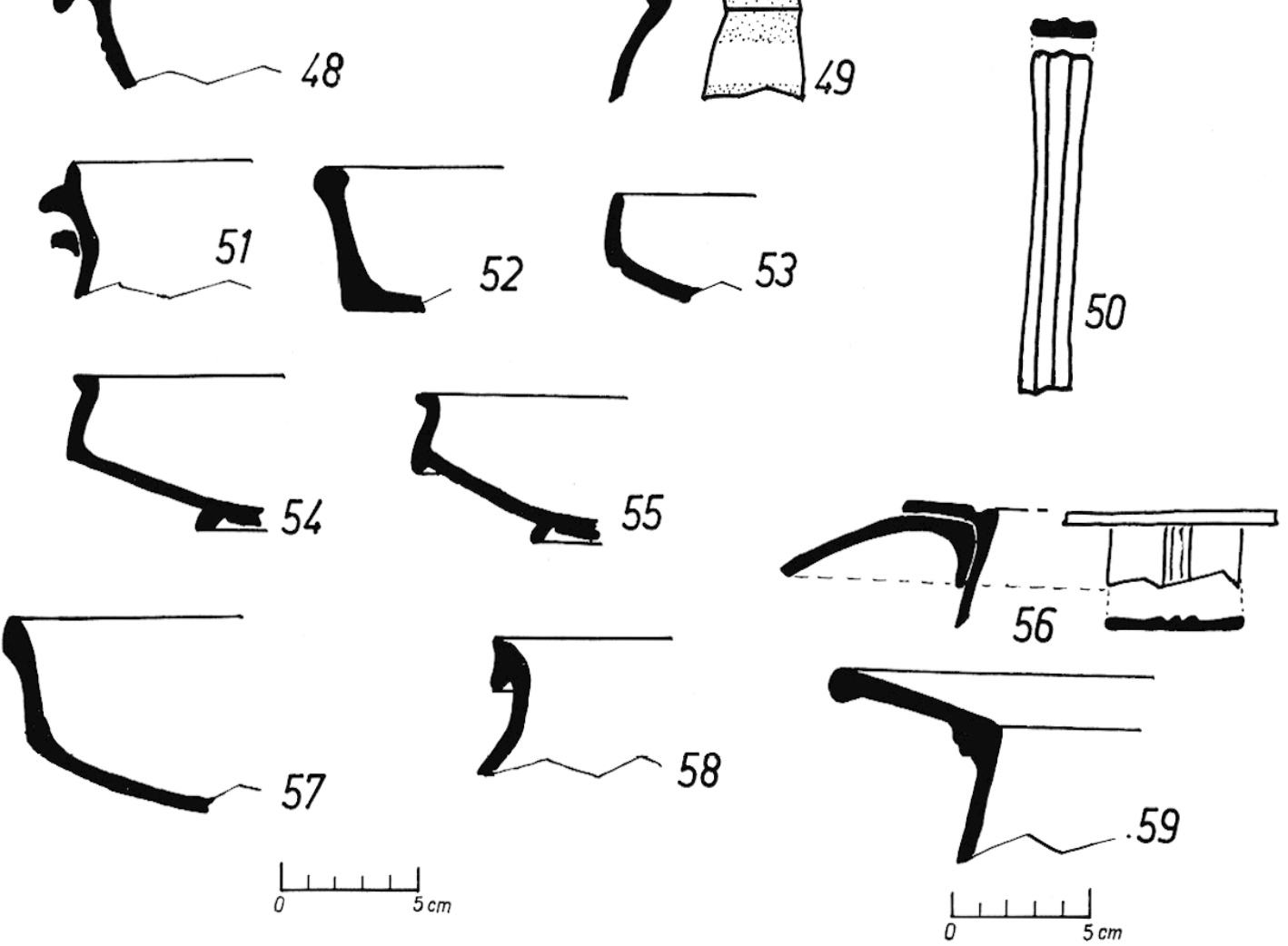

9 Les vases ou fragments nos 46,48 sont en terre sigillée et appartiennent à un contexte antérieur à la nécropole. Il en est de même pour l'anse de cruche blanche $n^{\circ} 50$. Les vases ou fragments nos 49 et 51 à 59 sont des offrandes de la nécropole.

grossière et présente de gros grains de quartz et de feldspath. Sa couleur est beige, la cuisson oxydante. Un enduit rouge a été passé sur toute la surface extérieure.

45. - Fragment d'amphore de forme Dressel 20. Non dessiné, mais important par sa présence. Il s'agit d'une anse qui ne présente pas de trace d'estampille.

49 (fig. 9). - Partie supérieure de petit vase ovoïde (?). Le col est prolongé par une lèvre en bourlet dont la partie supérieure est plate. La pâte est claire, de couleur bı ige et relativement fine. La surface extérieure montre que ce récipient a été recouvert de bandes blanches plus ou moins larges.

50 (fig. 9). - Anse de cruche fine et allongée appartenant vraisemblablement à un récipient assez haut. La pâte, très fine, est saumonnée et les surfaces sont recouvertes d'un engobe blanc.

51 (fig. 9). - Col de cruche à deux anses, cylindrique dans sa partie supérieure, une collerette vient s'ajouter extérieurement à la base du cylindre pour probablement servir de support à un bouchon? La pâte est beige, mi-fine, légèrement oxydée près des surfaces ; le cœur de la pâte reste sombre. Les surfaces sont rougeâtres. 
52 (fig. 9). - Assiette plate. Le flanc est évasé et légèrement déversé. La partie supérieure se termine par une lèvre en forme de large bourlet. La pâte est noire, très grossière. Les surfaces sont également d'un noir intense dû probablement à un engobe.

53 (fig. 9). - Petite coupe à rebord rentrant. Toute la partie inférieure manque. La pâte est mi-fine, de couleur gris-clair. Les surfaces sont d'un gris un peu plus sombre et montrent des traces de mica. L'ensemble est soigneusement lissé.

54 (fig. 9). - Coupe carénée. La panse forme une large courbe, peu incurvée. Elle se referme aux deux tiers environ de sa hauteur pour se terminer par un évasement qu'achève une lèvre dont le profil est triangulaire. A l'intersection des deux mouvements de la panse, on observe une sorte de redan. Le pied forme une couronne peu saillante. Le fond, séparé du pied par une gorge, est concave au dessous et, à l'intérieur, forme un ombilic saillant. Pâte et surfaces sont d'un beige assez sombre. La texture est mi-fine.

55 (fig. 9). - Coupe carénée. La panse forme une large courbe, peu incurvée. Elle se referme aux deux tiers environ de sa hauteur pour se terminer par un évasement qu'achève une lèvre à peu près triangulaire, nettement déversée. A l'intersection des deux mouvements de la panse, on observe une sorte de redan dont le profil forme un angle aigu. Le pied est en forme de couronne tronconique, peu saillant. La partie inférieure du fond est plate. La partie supérieure forme un léger ombilic. La pâte est grossière, de couleur beige.

56 (fig. 9). - Fragment de col et d'anse de très grand récipient. L'anse, très plate, est particulièrement large. Il s'agit d'une céramique très grossière, cuite en atmosphère oxydante. Sur

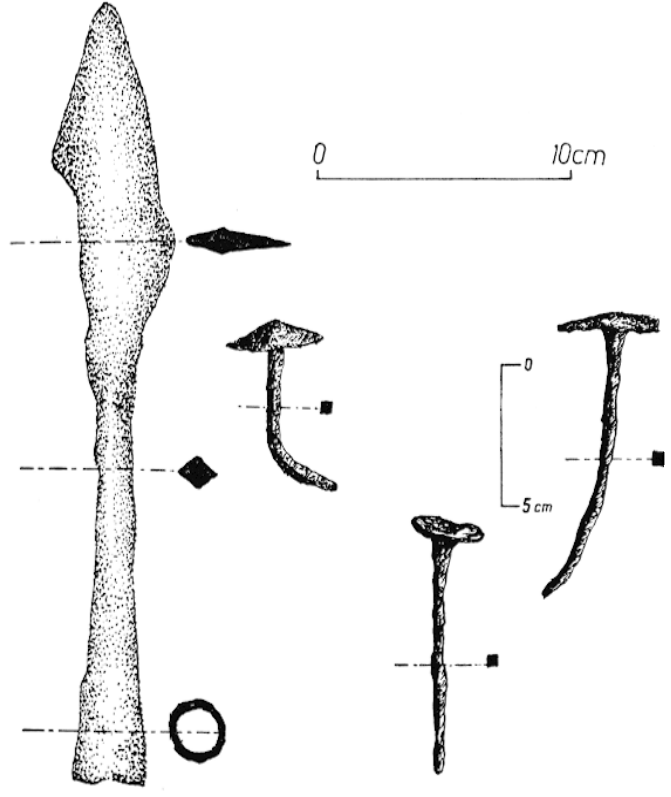

10 Fer de lance et clous. l'ensemble des surfaces, un engobe ou un enduit, d'un rouge très intense, a été passé.

57 (fig. 9). - Fragment de grande marmite tripode, évasée. Les pieds ont disparu mais leur trace est visible. La panse forme une carène et se termine, dans sa partie supérieure, par un bourlet extérieur. Le fond est dans le prolongement de la courbe de la panse. La pâte est grossière, noire. Les surfaces sont noires également.

58 (fig. 9). - Fragment de col de vase (ovoïde ?). La lèvre, en profil, a la forme d'une poulie. La pâte est noire, mi-fine. Les surfaces sont également noires ${ }^{62}$.

59 (fig. 9). - Fragment de col de grand récipient (proche du fragment $n^{0} 56$ ) à col évasé se terminant par un bourlet à l'extérieur. Il n'est pas possible de restituer la forme de la panse. La pâte est grossière, rougeâtre. Toutes les surfaces sont recouvertes d'un rouge (engobe ou peinture?) assez vif.

62 Ce type de col se rencontre sur des récipients en usage dans des gisements du III $^{\mathrm{e}}$ et du Iv $^{\mathrm{e}}$ siècle (cf. R. Périchon et Cl. Chopelin, La céramique estampée...). Il semble même que cet usage se prolonge bien au-delà (cf. Cl. Chopelin, La céramique du Moyen Age dans la région de Charlieu, public. du Groupe de Recherches archéologiques de Charlieu, 1969, pl. 1, fig. 6). 

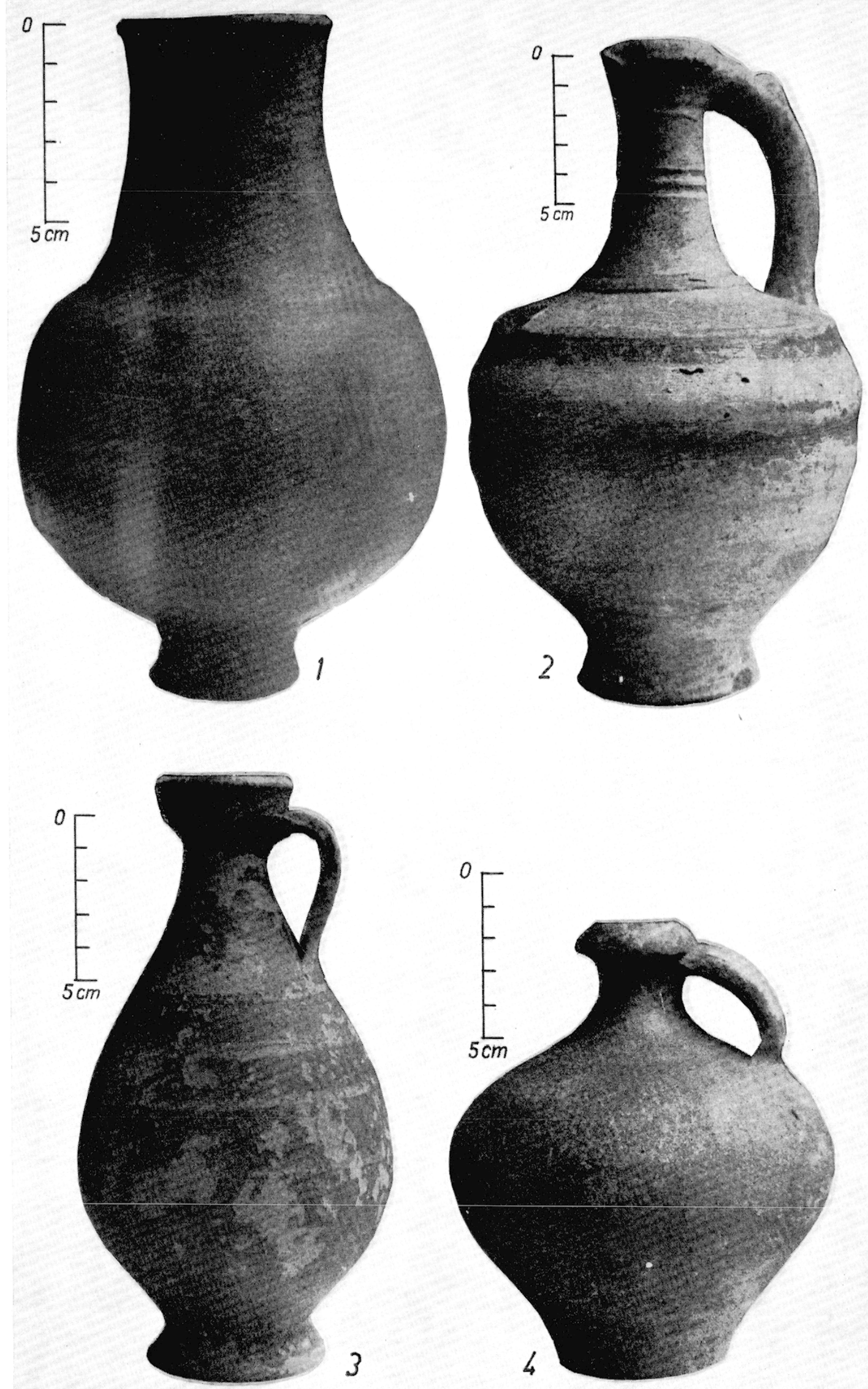

11 Céramiques communes, vases d'offrandes de la nécropole. 

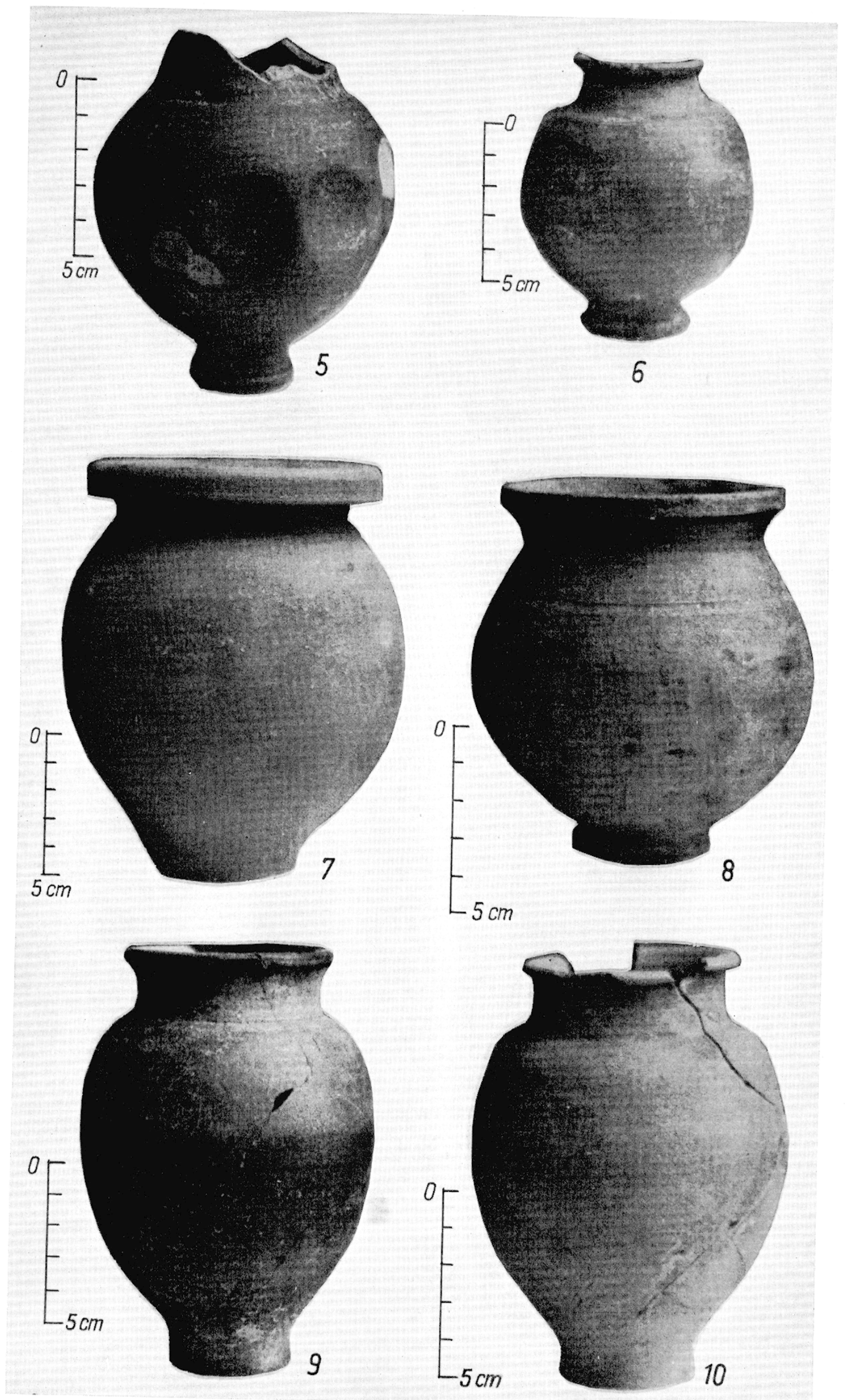

12 Céramiques communes, vases d'offrandes de la nécropole. 


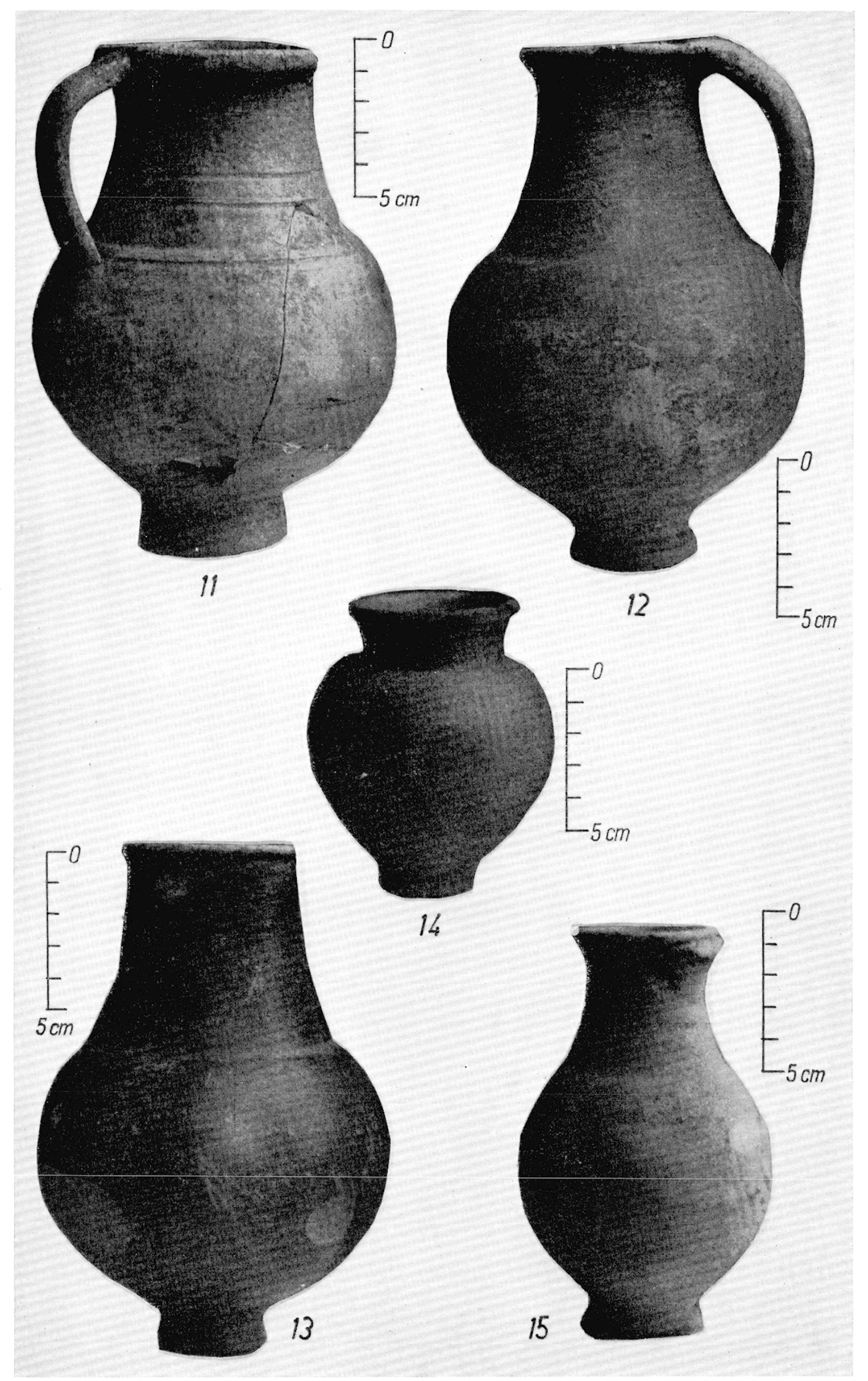

13 Céramiques communes, vases d'offrandes de la nécropole. 

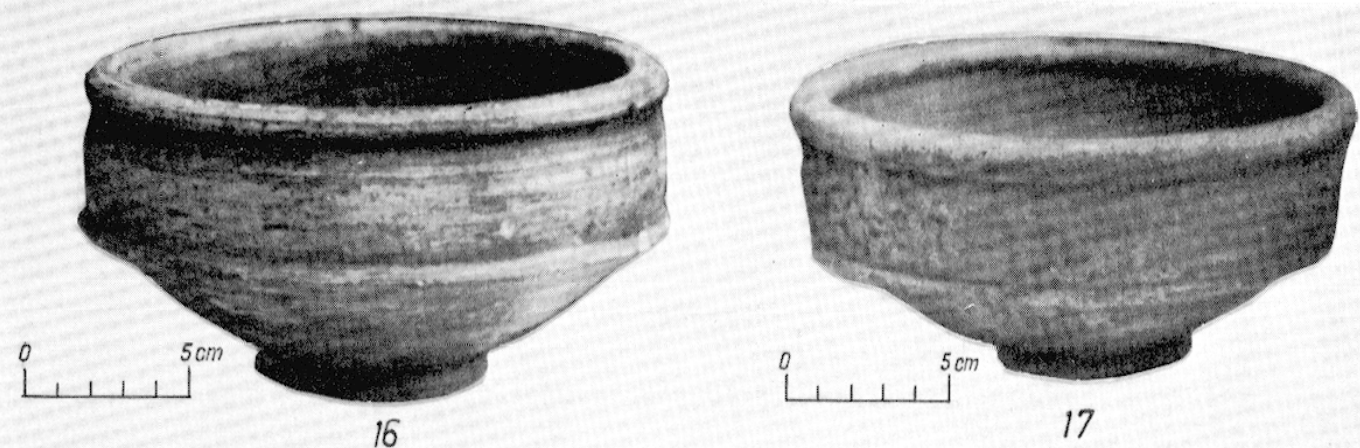

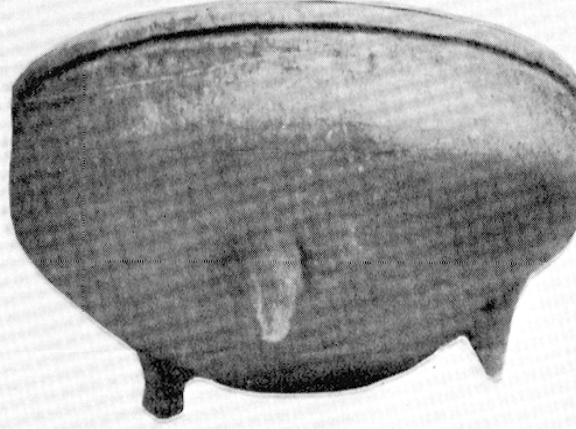

18

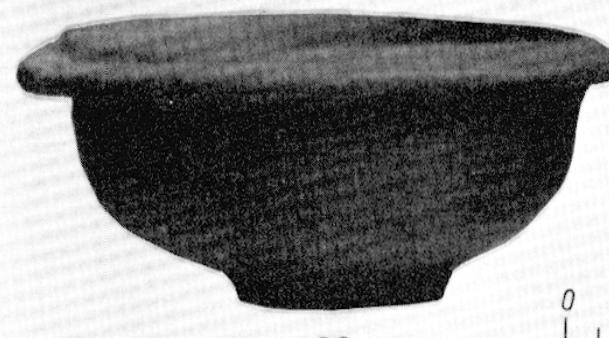

20

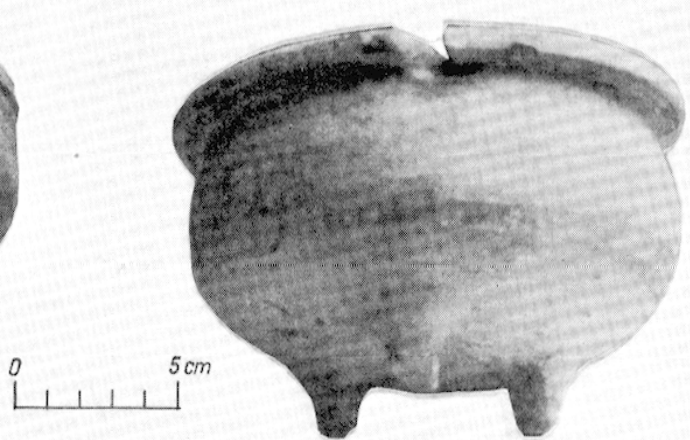

19

14 Céramiques communes, vases d'offrandes de la nácropole.

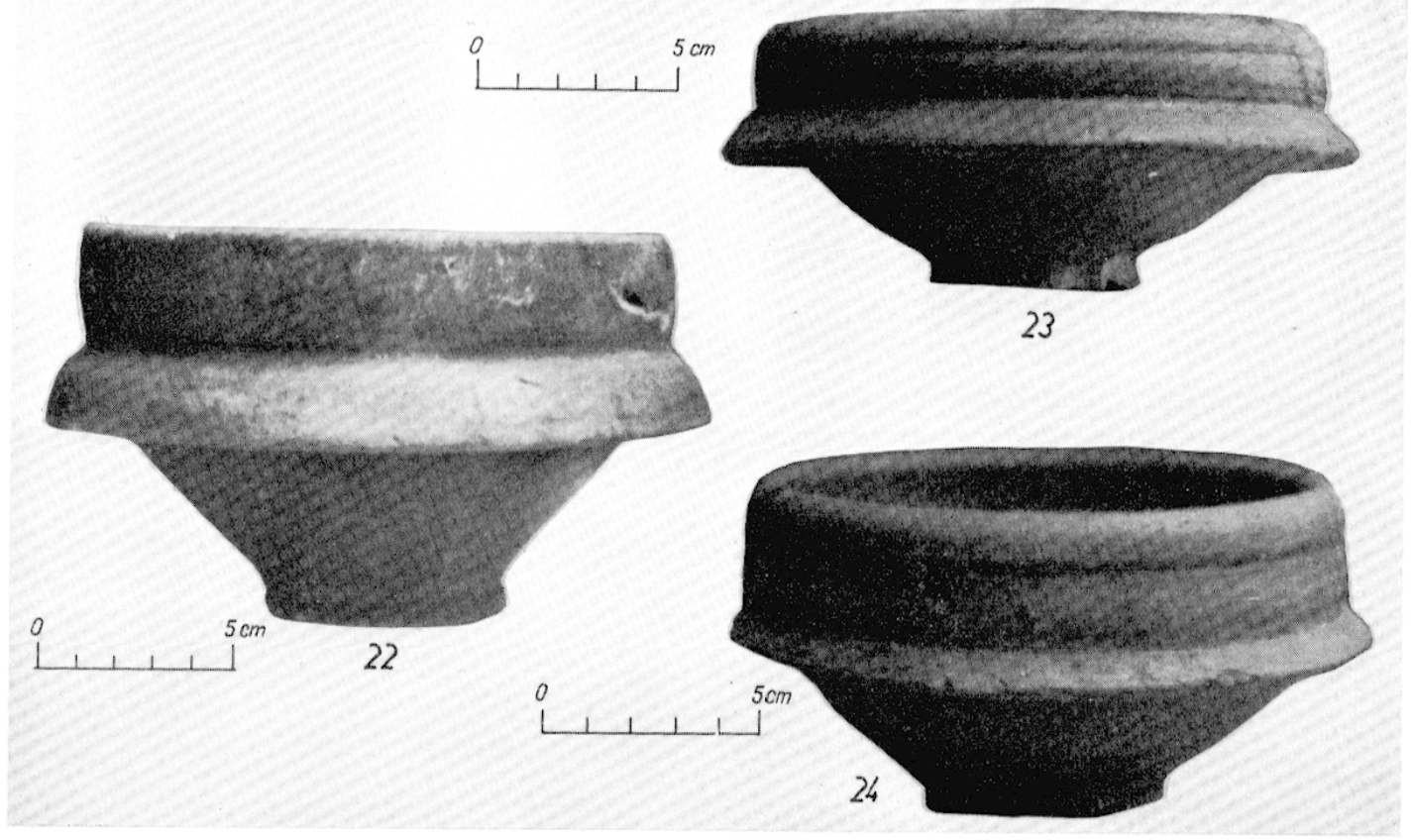

15 Céramiques communes, vases d'offrandes de la nécropole. 


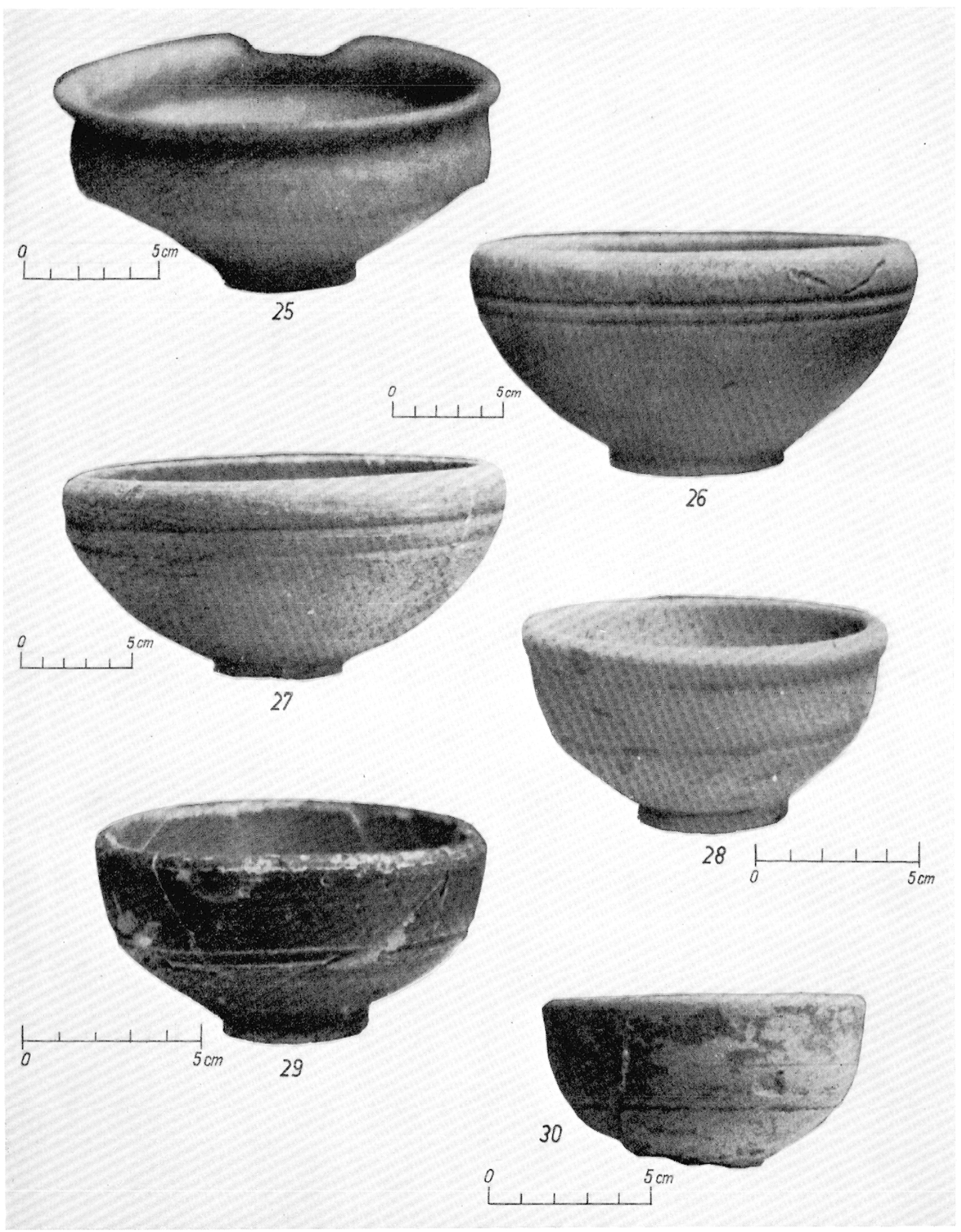

16 Les nos $25,26,27$ et 28 sont des céramiques communes. Les vases nos 29 et 30 sont des imitations de céramiques sigillées. 


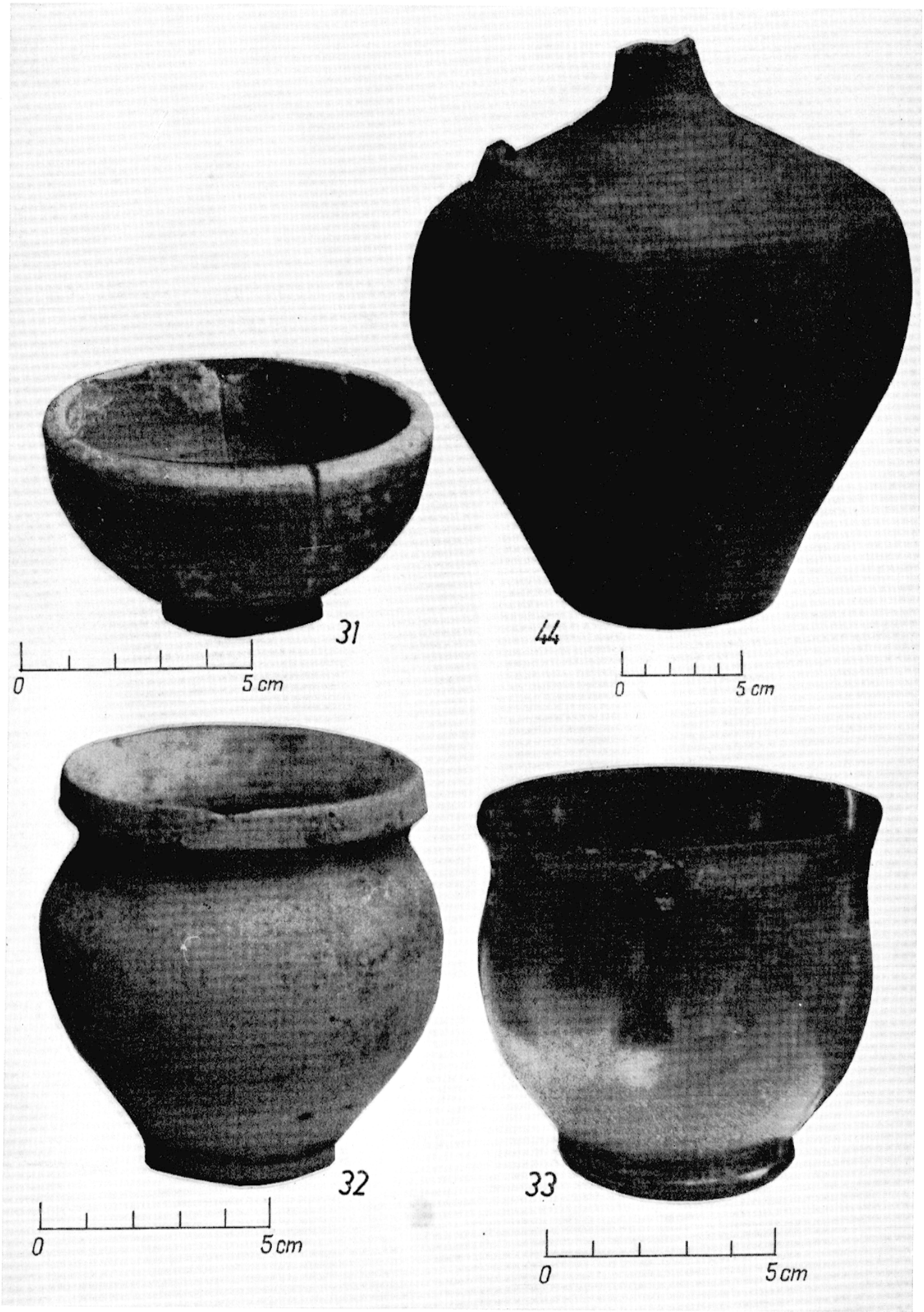

17 Les nos 32 et 44 sont des céramiques communes. Le no 31 est une petite coupe imitant la céramique sigillée. Le $n^{\circ} 33$ est un récipient en verre. 
III. Objets en métal.

60 (fig. 10). - Fer de lance, assez bien conservé. La lame présente un profil losangique obtenu à partir d'un saumon de profil carré encore visible dans la partie qui sépare cette dernière de la douille. Ce genre d'arme a été utilisé par des peuples très différents, en conséquence, il est difficile de lui attribuer une origine. Notons que ce fer a été trouvé en dehors de la nécropole et que, par conséquent, il ne constituait pas une offrande.

61 (fig. 10). - Clous en fer. Ces clous se présentent parfois avec de larges têtes. Leur quantité semble minime par rapport au reste du mobilier. Leur facture manuelle est indéniable. Nous ne pensons pas qu'il s'agisse de clous de cercueils mais rien ne nous permet de préciser une autre utilisation.

IV. Le verre.

33 (fig. 8 et 17). - Petit bol à pied. La panse est arrondie et se termine par un col évasé dont la lèvre est coupée. Le pied est en couronne, tronconique. Le fond, issu de la couronne du pied, est particulièrement bombé à l'intérieur. Le récipient est de couleur verdâtre, presque blanc. Il ne présente aucune décoration.

Robert Périchon et Glaude Ghopelin 